\title{
Transport Phenomena: Challenges and Opportunities for Molecular Catalysis in Metal-Organic Frameworks
}

\author{
Ben A. Johnson, Anna M. Beiler, Brian D. McCarthy, and Sascha Ott* \\ Cite This: J. Am. Chem. Soc. 2020, 142, 11941-11956 \\ Read Online
}

ABSTRACT: Metal-organic frameworks (MOFs) are appealing heterogeneous support matrices that can stabilize molecular catalysts for the electrochemical conversion of small molecules. However, moving from a homogeneous environment to a porous film necessitates the transport of both charge and substrate to the catalytic sites in an efficient manner. This presents a significant challenge in the application of such materials at scale, since these two transport phenomena (charge and mass transport) would need to operate faster than the intrinsic catalytic rate in order for the system to function efficiently. Thus, understanding the fundamental kinetics of MOF-based molecular catalysis of electrochemical reactions is of crucial importance. In this Perspective, we quantitatively dissect the interplay between the two transport phenomena and the catalytic reaction rate by applying models from closely related fields to MOF-based catalysis. The identification of the limiting process provides opportunities for optimization that are uniquely suited to MOFs due to their tunable molecular structure. This will help guide the rational design of efficient and high-performing catalytic MOF films with incorporated molecular catalyst for electrochemical energy conversion.

\section{INTRODUCTION}

Metal-organic frameworks (MOFs) are emerging as widely popular support structures to incorporate molecular catalysts for the electrochemical activation and conversion of small molecules. ${ }^{1-3}$ Composed of discrete molecular building blocks (organic linkers and metal nodes), ${ }^{4,5}$ MOFs have a wide range of synthetic tunability. ${ }^{6,7}$ Varying the microscopic molecular structure produces materials with different pore sizes and chemical environments, perhaps allowing for augmentation of the active site of molecular catalysts. ${ }^{8}$ In addition, the permanent porosity and high internal surface area of many MOFs could result in very high active-site densities, ${ }^{9}$ which is necessary for the fast transformation of substrate into product per unit volume. $^{10}$

However, when moving away from a homogeneous environment to heterogeneous porous films, chemical reactions become interfacial in nature as a consequence of immobilizing or heterogenizing the molecular catalyst into a three-dimensional support matrix. For the system to operate efficiently in such cases, both charge and substrate must be transported to the catalytic sites within the film at a faster rate than the intrinsic turnover rate of the catalytic reaction. We postulate in this Perspective that one critical, but often overlooked, aspect of utilizing MOFs for the molecular catalysis of electrochemical reactions is transport.

In MOF films with discrete redox-active molecular linkers, electron-hopping-formally governed by Fick's laws of diffusion-delivers charge to the catalytic sites. Therefore, the diffusional transport of both charge and substrate needs to be appropriately accounted for in order to quantitatively characterize and optimize each potentially limiting process. Diffusion has the potential to be much slower compared to other processes occurring during catalysis, particularly chemical reaction rates, and this can be exacerbated in the confined environments of porous materials. Given the microporous nature of MOFs, transport phenomena are expected (and in a few exceptional cases have been qualitatively shown, as will be discussed) to have an enormous effect on the overall catalytic performance of electroactive MOF films. The observed reaction rate easily can be limited by mass or charge transport if these phenomena are not controlled or optimized. While experimentally examining MOF-based reactions, it is imperative to remember that when these reactions are coupled with diffusion, ${ }^{11,12}$ substrates and/or catalytic intermediates will have spatially dependent concentration profiles, and their concentration throughout the film may not reflect the bulk concentrations of these species.

In the following sections, we will explore well-established electrochemical methods to quantify rates of charge transport, substrate diffusion, and the intrinsic catalytic reaction. Next, the question, "Where is the bottleneck for catalysis?" is posed and answered by examining each potentially limiting transport process in detail with numerical simulations, incorporating relevant data from the literature. Concepts of charge transport dynamics and catalytic reaction engineering from closely related fields are utilized to understand these issues on a fundamental level. Finally, looking to the future, the quantitative analysis and parameters detailed in the beginning sections are used to understand how to optimize catalytic performance and design new MOFs, which alleviate transport limitations. The reaction-

Received: March 13, 2020

Published: June 9, 2020 
diffusion kinetic models outlined here reveal important design rules, and indicate which features of the catalyst-support system to select and optimize in order to rationally control transport and ultimately reactivity. While the incorporation of transport phenomena into kinetic studies of MOF-based catalysis is under-represented in the literature, it is certainly not unique to MOFs. Many of the concepts of reaction-diffusion kinetics that will be discussed here apply widely to other micro- or mesoporous catalyst materials. ${ }^{10,13-15}$ However, MOFs do offer a unique opportunity for rational control over mass and charge transport properties at the molecular level. This is enabled by the vast array of combinations for both the organic linkers and the connectivity between these building blocks via the inorganic nodes, as well as the synthetic tuning possible for both these functionalities. More research is certainly needed to translate molecular-level design of MOFs into targeted control over macroscopic transport properties, which is crucial to optimize catalysis for large-scale applications. However, the first step toward this goal, and consequently the focus of the following discussion, is to delineate and quantitatively characterize the influence of transport phenomena on chemical reactivity in MOFs by simple kinetic models, leading to specific design criteria for not only transport properties but also for important extrinsic parameters such as film thickness. This has implications for MOFs applied to the electrochemical conversion of small molecules for energy related processes (fuel-forming reactions, water oxidation etc.), but also potentially to recently revitalized electrochemical organic transformations and redox catalysis producing high-value organic products. ${ }^{16-18} \mathrm{We}$ end by summarizing the opportunities and challenges for MOFs entering applications as high-performance catalytic films for this variety of possible electrochemical reactions.

Revisiting Reaction-Diffusion. The analysis of transport phenomena occurring in porous media and their effect on catalytic reactions has some important historical origins. The year 2019 celebrated the 80th anniversary of Prof. E. W. Thiele's pioneering publication, "Relationship between Catalytic Activity and Size of Particle". ${ }^{19}$ Therein, Thiele presented a model for reaction-diffusion occurring in porous catalyst particles or "grains" and outlined two regimes: (1) where the intrinsic kinetics of the catalyst solely limit the overall reaction rate, and (2) where internal substrate diffusion limits the overall reaction rate. An important part of these results accounts for the transition between these two scenarios in a quantitative manner and predicts the resulting behavior based on a small number of input parameters. This is accomplished by defining a single descriptor, a dimensionless control parameter that has come to be known as the Thiele modulus $\phi$,

$$
\phi=R_{\mathrm{a}} \sqrt{\frac{k_{\mathrm{cat}}}{D_{\mathrm{S}}}}
$$

where $R_{\mathrm{a}}$ is the radius of the equivalent sphere, $k_{\mathrm{cat}}$ is the firstorder rate constant of the catalytic reaction, and $D_{S}$ is the diffusion coefficient of the substrate inside the particle. This very simply states that the efficiency of any chemical reaction occurring in concert with diffusion will be a function of a geometric length scale describing the physical system, the catalytic rate, and the diffusivity of the substrate. If the time scale required for substrate to diffuse through the particle is large compared to the reaction rate $(\phi>1)$, the substrate will be consumed by the reaction before permeating through the entire particle. A boundary layer (also referred to as a reaction- diffusion layer) forms near the particle surface, which limits the amount of catalyst actively participating in the reaction-in other words, the catalysts in the interior of the particle are largely dormant (Figure 1). Thus, porous catalysts with large particle
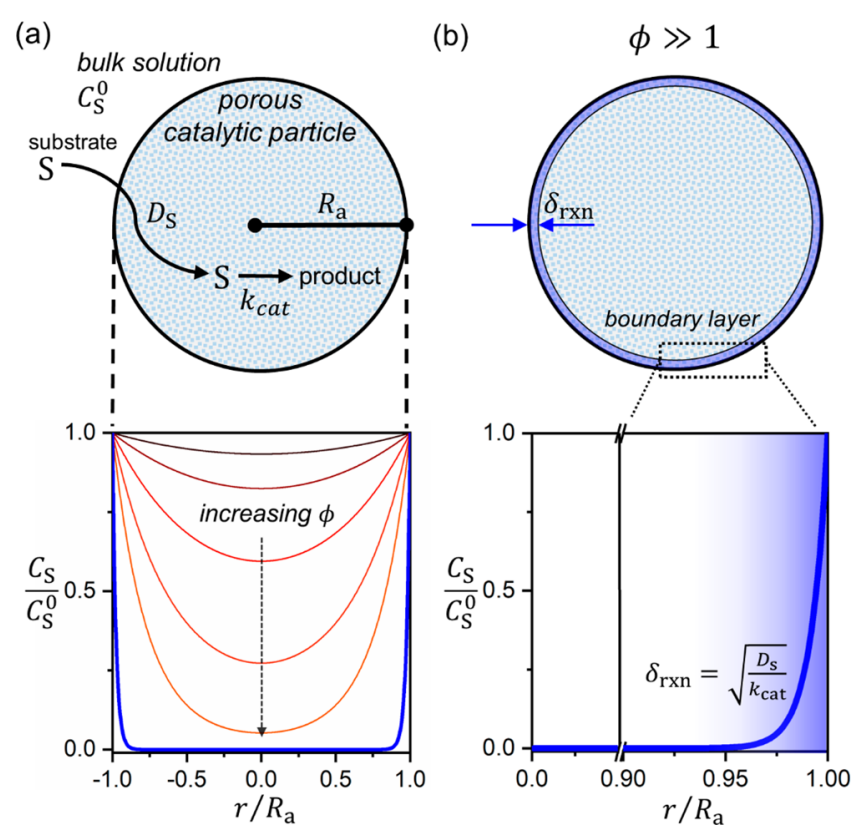

Figure 1. (a) Quantitative representation of a boundary or reactiondiffusion layer in a MOF particle (approximated as spherical) for a firstorder reaction at steady state. Dimensionless concentration is displayed on the $y$-axis, where $C_{\mathrm{S}}$ is the substrate concentration inside the MOF particle (a function of the radial distance $r$ ), and $C_{S}^{0}$ is the bulk substrate concentration (taken as constant outside the particle for simplicity). The dimensionless radial coordinate $\left(R_{\mathrm{a}}\right.$ is the particle radius) is plotted on the $x$-axis. The arrow indicates increasing values of the Thiele modulus, $\phi>1$. (b) Expanded region showing reaction-diffusion layer, with thickness given by $\delta_{\text {rxn }}$.

sizes and/or very fast reactions will have a significant diffusional resistance that lowers the observed reaction rate. ${ }^{13,20,21}$ Therefore, an optimized catalytic material will inevitably have these parameters balanced appropriately, so that any limitations of transport by diffusion on the reaction rate are small or negligible. Thiele developed his model for catalyst particles of several different geometries, but the same underlying physical principle applies here to porous MOF film electrodes. In either case, overall efficiency is controlled by the ratio of the intrinsic catalytic reaction rate to a particular diffusion rate (this could be the diffusion of either charge or substrate through the MOF film). Efficiency, in this context, denotes how much of the catalyst is utilized during the reaction in the particle or film, i.e., the ratio of the amount of active catalyst to the total amount of catalyst contained in the MOF. This metric is canonically referred to as the "effectiveness factor" ${ }^{21}$ and is easily quantified as the observed reaction rate divided by the maximum reaction rate in the absence of any diffusional gradients or transport limitations. ${ }^{13,20,21}$ Practitioners in MOF-based catalysis of both chemical and electrochemical reactions are encouraged to read Thiele's original paper-its message is indeed still as timely now as it was in 1939. We will see in later sections how the same physical principles captured by Thiele's modulus can influence catalysis of electrochemical reactions mediated by molecular species covalently incorporated in MOF film electrodes. For convenience, the dimensionless parameters used in this 
Table 1. Dimensionless Parameters ${ }^{a}$

\begin{tabular}{cc}
\hline symbol & $R_{\mathrm{a}} \sqrt{\frac{k_{\mathrm{cat}}}{D_{\mathrm{S}}}}=\left(\frac{\text { reaction rate }}{\text { diffusion rate }}\right)=\left(\frac{\text { particle size }}{\text { reaction-diffusion layer thickness }}\right)$ \\
$\lambda_{\mathrm{e}}$ & $d_{\mathrm{f}} \sqrt{\frac{F v}{D_{\mathrm{e}}^{\text {app }} R T}}=\left(\frac{\text { film thickness }}{\text { diffusion layer thickness }}\right)$ \\
$\Lambda_{\mathrm{s}}$ & $k_{\mathrm{s}} \sqrt{\frac{R T}{D_{\mathrm{e}}^{a p p}} F v}=\left(\frac{\text { interfacial electron transfer rate }}{\text { voltammetric diffusion rate }}\right)$ \\
$\sqrt{\frac{i_{\mathrm{k}}}{i_{\mathrm{s}}}}$ & $d_{\mathrm{f}} \sqrt{\frac{k C_{\mathrm{P}}^{0}}{D_{\mathrm{S}}}}=\left(\frac{\text { reaction rate }}{\text { substrate diffusion rate }}\right)=\left(\frac{\text { film thickness }}{\text { reaction-diffusion layer thickness for substrate }}\right)$ \\
$\sqrt{\frac{i_{\mathrm{k}}}{i_{\mathrm{e}}}}$ & $d_{\mathrm{f}} \sqrt{\frac{k \kappa_{\mathrm{S}} C_{\mathrm{s}}^{0}}{D_{\mathrm{e}}^{\mathrm{app}}}}=\left(\frac{\text { reaction rate }}{\text { charge transport diffusion rate }}\right)=\left(\frac{\text { film thickness }}{\text { reaction-diffusion layer thickness for charge transport }}\right)$
\end{tabular}

${ }^{a}$ A full list of all dimensional symbols and their definitions is given in the Symbols section.

Perspective are summarized in Table 1. It is important to highlight the outstanding independent contribution of Dr. Gerhard Damköhler, who arrived at the same relationship between mass transport and chemical reactions. ${ }^{22}$ In his honor, the Damköhler number $(\mathrm{Da})$ is often used to represent the ratio between a particular reaction rate and a convective mass transport rate. $^{21}$

\section{CHARGE TRANSPORT DYNAMICS}

Returning to electroactive MOF films, in this section we will introduce two primary microscopic charge transport mechanisms, band or ohmic conduction (migration) and electronhopping conduction (formally diffusion), which carry electrons or holes to the covalently incorporated molecular catalysts. MOFs with discrete redox-active linkers (e.g., molecular metallocatalysts) most often exhibit an electron-hopping mechanism, which will be the main focus of the following discussion. Since electron hopping is formally a diffusion process (vide infra), these concepts will become important when considering the reciprocity between the rate of charge transport and catalysis and how the resulting reaction-diffusion behavior of the film can affect catalytic efficiency and kinetic measurements.

Band or ohmic conduction occurs when the electronic states of the MOF are highly delocalized and possibly strongly coupled to the electrode's electronic states, due to through-bond or through-space conjugation of the organic linkers and/or metal nodes. ${ }^{23}$ Electron movement in the film in this case is driven by migration as a result of electrical potential gradients. Experimentally, cyclic voltammograms (CVs) of such films display only capacitive current (faradaic waves are only observed if interaction with a substrate molecule causes the injected charge to be localized at a specific site). ${ }^{24,25}$ An example of this behavior was demonstrated by a highly conjugated (electrical conductivity $\sigma=40 \mathrm{~S} \mathrm{~cm}^{-1}$ ) $\mathrm{Ni}_{3}$ (hexaiminotriphenylene) ${ }_{2} 2$-D MOF reported by Dincă and co-workers for the oxygen reduction reaction (ORR). ${ }^{26}$ Under a $\mathrm{N}_{2}$ atmosphere, a large capacitive wave is observed, while conversely if $\mathrm{O}_{2}$ is added to the cell, there is a clear increase in current corresponding to the faradaic signature of the charge transfer reactions occurring during catalysis.
Alternatively, a MOF film may have components that are redox-active within a given potential window but are not electronically coupled to one another through conjugation, where the electronic states of the MOF are now spatially localized and defined by standard potentials $\left(E^{0}\right)$. Thus, in this instance electron hopping will be the main charge transport mechanism, and electron movement is driven by a series of bimolecular self-exchange electron transfer reactions between the reduced form of the linker or node and the oxidized form of the linker or node in close proximity. ${ }^{27}$ The voltammetric response of this type of MOF film exhibits faradaic waves even in the absence of substrate and has been characterized in many recent examples. ${ }^{28-34}$

Dahms ${ }^{35}$ and Ruff ${ }^{36-38}$ first outlined the theory for electron transport via self-exchange reactions, including a contribution for the local physical displacement of the redox-active molecules. Andrieux and Savéant, ${ }^{27}$ as well as Laviron, ${ }^{39}$ developed an extension of this initial work by considering only stationary or fixed sites (most relevant to MOFs), with significant experimental contributions from Murray, ${ }^{40}$ Anson, ${ }^{41}$ and Bard, ${ }^{42,43}$ utilizing redox-polymer-modified electrodes. By definition, there is zero driving force for a self-exchange reaction; however, an activation barrier still is present, and a rate constant $k_{\mathrm{ex}}$ can be defined for the reaction between linkers in adjacent layers (Figure 2). For a given geometry, each electron "hop" occurring in a random direction is governed by a

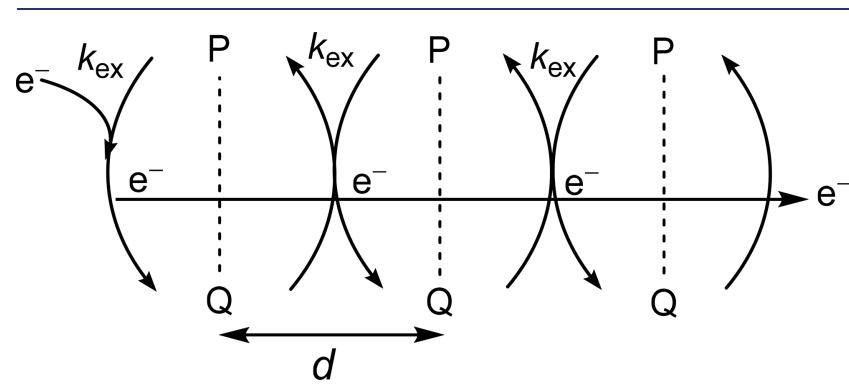

Figure 2. Electron-hopping mechanistic scheme for the reaction $\mathrm{P}+\mathrm{e}^{-}$ $\rightleftharpoons \mathrm{Q}$ in a redox-active MOF film occurring between two layers, separated by an average hopping distance $d$. 
time-step set by $k_{\mathrm{ex}}$. The overall process is in essence a random walk, and therefore can be formally modeled by the diffusion of fixed redox-active centers. Electron self-exchange or "hopping" through a molecular redox film responds to concentration gradients or, more accurately, chemical potential gradients created at the electrode surface by the applied potential. ${ }^{40,44}$ Thus, similar to a freely diffusing species in solution, the change in concentration of each linker in a particular oxidation state within the film formally adheres to Fick's laws.

An equivalent diffusion coefficient $D_{\mathrm{e}}$ for this electronhopping mechanism was defined ${ }^{27,39}$ and is shown in eq 2 ,

$$
D_{\mathrm{e}}=\frac{k_{\mathrm{e}}}{6} C_{\mathrm{P}}^{0} d^{2}
$$

where $C_{\mathrm{P}}^{0}$ is the total redox-active linker concentration, $d$ is the average hopping distance, normally taken as the distance between nearest neighbors in the lattice, and $k_{\mathrm{e}}$ (with $k_{\mathrm{e}}=$ $\left.6 k_{\mathrm{ex}}\right)$ is the electron-transfer rate constant assuming selfexchange occurs only in the direction normal to the electrode surface with, however, six possible nearest-neighbor sites. The value $\left(k_{\mathrm{e}} / 6\right) C_{\mathrm{P}}^{0}$ is sometimes referred to as " $k_{\text {hopping }}$ " $\left(\mathrm{s}^{-1}\right){ }^{28,29,45}$

Counterion Diffusion-Migration. Further refinement of the above model takes into account that electron displacement is associated with the movement of counterions to maintain electroneutrality. ${ }^{46-48}$ Transient measurements (potential-step chronoamperometry) include macroscopic diffusion of chargebalancing mobile counterions, as a net flow of electrons is concomitant with a net displacement of mobile counterions within the film and across the film-solution interface, resulting in an apparent diffusion coefficient, $D_{\mathrm{e}}^{\text {app }}$. Notably, Cottrell behavior has been repeatedly observed for these systems (the transient current after a large potential step is proportional to $\left.t^{-1 / 2}\right) .^{28-30,34,49-51}$

It has been recently demonstrated ${ }^{44}$ that if the overall process involves ion-coupled electron transfer reactions (where an electro-inactive counterion participates in the self-exchange reaction), the maximum conductivity of the charge transport process will be dominated by the slower of two processes, characterized by either a purely ionic diffusion coefficient for mobile counterion displacement or an apparent diffusion coefficient for the ion-coupled charge-hopping process. ${ }^{44}$ This may well be the case in MOFs, as reported recently by Morris and co-workers, where the purely ionic diffusion coefficient and the electron-hopping diffusion coefficient were independently determined for charge transport between ferrocene units anchored in NU-1000. ${ }^{31}$ In any case, the microscopic interpretation of $D_{\mathrm{e}}^{\text {app }}$ will be highly dependent on the microscopic charge transport mechanism or model. ${ }^{40}$

Additionally, significant electrostatic interactions such as ionpairing may exist within MOF pores. In a theoretical treatment of charge hopping in redox polymers, which have relatively nonpolar interiors with potentially very high concentrations of ionic species, it was shown that $D_{\mathrm{e}}^{\text {app }}$ decreases with increasing association constant for the ion-paired species between the redox-active component in the polymer backbone and the mobile redox-inactive counterion. ${ }^{52,53}$ The effect of ion-pairing on charge transport in MOFs has recently been examined, and initial results support the dependence of $D_{\mathrm{e}}^{\text {app }}$ on the ion-pairing association constant. ${ }^{31}$

Cyclic Voltammetric Analysis of Electroactive MOF Films. Applying these theoretical treatments, it has been established that macroscopic electron transport through MOF films with discrete redox-active components proceeds formally as a diffusion process. ${ }^{50,54}$ The voltammetric response of such a MOF film will therefore be very similar to that of a freely diffusing species in solution, however, with the very important difference that the formal diffusion of fixed redox-active linkers is occurring within a finite domain. This means the boundary condition at the solution-film interface needs to be considered and will give rise to both linear finite and semi-infinite diffusional responses depending on the rate of diffusion relative to the time scale of the voltammogram (set by the scan rate in CV) and the film thickness. Further, if electron transfer between the electrode and the first layer in the film is unconditionally at equilibrium during the scan, the boundary condition at the electrode-film interface obeys the Nernst equation. Alternatively, ButlerVolmer kinetics can describe the electron transfer reaction occurring at the electrode surface if the reaction is slow relative to the CV time scale. Transition between these complementary behaviors and the overall current-potential response are captured by two dimensionless parameters, originally defined by Matsuda and co-workers ${ }^{55,56}$ and Savéant and co-workers: ${ }^{27}$ the finite diffusion parameter, $\lambda_{\mathrm{e}}$, and the kinetic parameter, $\Lambda_{\mathrm{s}}$, given below,

$$
\begin{aligned}
& \lambda_{\mathrm{e}}=d_{\mathrm{f}} \sqrt{\frac{F \nu}{D_{\mathrm{e}}^{\mathrm{app}} R T}} \\
& \Lambda_{\mathrm{s}}=k_{\mathrm{s}} \sqrt{\frac{R T}{D_{\mathrm{e}}^{\mathrm{app}} F \nu}}
\end{aligned}
$$

where $d_{\mathrm{f}}$ is the film thickness, $\nu$ is the scan rate, $D_{\mathrm{e}}^{\mathrm{app}}$ is the electron-hopping diffusion coefficient, $F$ is the Faraday constant, and $k_{s}$ is the standard rate constant for heterogeneous electron transfer. The competition between diffusion and interfacial electron transfer is expressed by $\Lambda_{\mathrm{s}}$, and $\lambda_{\mathrm{e}}$ is the ratio of the film thickness to the diffusion layer thickness. First, taking limits of the finite diffusion parameter under Nernstian electrode kinetics $\left(\Lambda_{\mathrm{s}}>1\right)$, when $\lambda_{\mathrm{e}} \rightarrow \infty$, the diffusion layer thickness, over which there is a significant concentration gradient of reduced/oxidized linkers, becomes much smaller than the film thickness (thick films, fast scan rates). As a result, electron-hopping diffusion can be approximated as taking place in a semi-infinite domain. The current-potential response is a classical diffusion wave (Figure $3 \mathrm{a}$; for examples of the concentration profiles of the reduced linker during the scan, see the Supporting Information) with a peak separation of $57 \mathrm{mV}$ and peak current proportional to $\sqrt{\nu}$. In the opposite limit of thin films or slow scan rates, as $\lambda_{\mathrm{e}} \rightarrow 0$, the same material will display zero peak separation and peak current proportional to $\nu$, characteristic of a symmetric "adsorption" or "surface" wave (Figure 3c). Here, the diffusion layer becomes large and starts to explore the finite geometry imposed by the film thickness-a situation of diffusion in a finite domain. Figure $3 \mathrm{~b}$ shows an intermediate regime with $\lambda_{\mathrm{e}}=2$. A crucial feature of this analysis is that even if a symmetric adsorption wave is observed, the electron transport mechanism occurring through the MOF film is still formally diffusional with, however, a finite boundary condition representing the film-solution interface. Simulations of CVs demonstrating the two behaviors with different scan rates can be found in the Supporting Information. Slow interfacial electron transfer at the electrode-film interface $\left(\Lambda_{s}<1\right)$ gives rise to larger peak potential separation in either limit of $\lambda_{\mathrm{e}}$, expected for electrochemical irreversibility (shown in the blue CVs in Figure 3, $\Lambda_{s}=0.2$ ).

As expressed by $\lambda_{\mathrm{e}}$, the transition between these two behaviors will occur on variation of either the scan rate or the film 


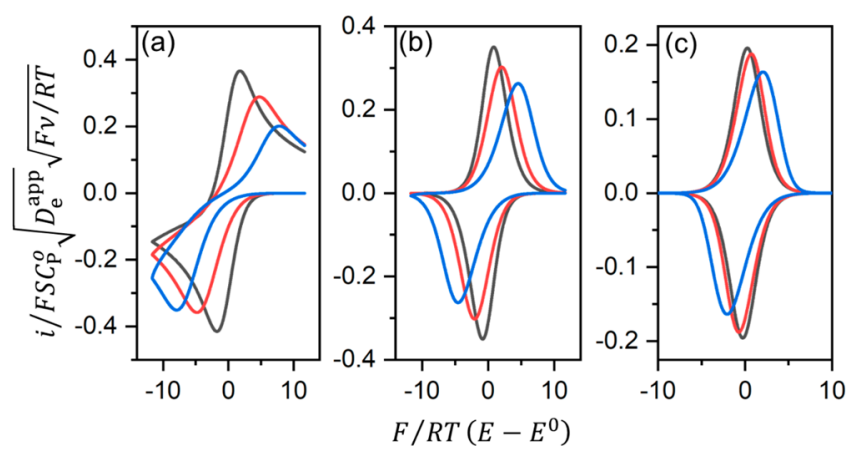

Figure 3. Simulated CVs of a molecular redox film using a range of values for the finite diffusion parameter $\lambda_{\mathrm{e}}:(\mathrm{a})=7$, (b) 2, and (c) 0.7, each with varying magnitudes of the heterogeneous electron transfer parameter $\Lambda_{s}$ (black, 10 ; red, 1 ; blue, 0.2 ). Current $i$ is normalized to the semi-infinite current $\left(F S C_{\mathrm{P}}^{0} \sqrt{D_{\mathrm{e}}^{\mathrm{app}}} \sqrt{F \nu / R T}\right)$. The normalized electrode potential $F / R T\left(E-E^{0}\right)$ is plotted on the horizontal axis. Current-potential responses were generated numerically with finite difference method.

thickness, approximately when $\lambda_{\mathrm{e}}$ is equal to unity. Pushing the system from one regime to the other will provide a method to extract the diffusion coefficient, if the film thickness can be measured independently (e.g., SEM cross section imaging). For example, in a recent report from our lab, a Zr-based MOF film containing molecular Co catalysts (UU-100) showed that the $\mathrm{CV}$ peak current transitioned from proportionality with $\nu$ to proportionality with $\sqrt{\nu}$ at $\sim 100 \mathrm{mV} \mathrm{s}^{-1}$. ${ }^{32}$ Since the film thickness was known, it was possible to estimate $D_{\mathrm{e}}^{\mathrm{app}}$ as being on the order of $10^{-8} \mathrm{~cm}^{2} \mathrm{~s}^{-1}$, which matched well with the value obtained from potential-step chronoamperometry experiments. It should be noted that the CVs displayed in Figure 3 do not account for non-idealities such as significant intermolecular interaction between linkers that would lead to broadening of the faradaic waves, ${ }^{57,58}$ which may be encountered in the case of many MOF-based electrodes.

Deviations from the CVs in Figure 3, for example in the observed peak current, could also occur as a result of inhomogeneous film thickness (roughness). ${ }^{59,60}$ A method developed recently by Buesen et al. delivers a way to determine the film thickness under operating conditions (with the film in the solvated state). ${ }^{60}$ The authors demonstrated that deviations from the ideal behavior described above could be used to estimate film roughness, quantified as an ensemble thickness distribution. ${ }^{59}$ These are important parameters to measure and optimize (surface roughness, film thickness, and charge transport diffusion coefficients), since they are tied to the reaction-diffusion behavior of the system, as demonstrated by the Thiele modulus, and inevitably control catalytic performance, which we will see in the following sections.

\section{MOLECULAR CATALYSIS IN MOF-BASED ELECTRODES}

The occurrence of diffusional transport of both charge and substrate presents a significant challenge in extracting kinetic parameters (rate constants or turnover frequencies, TOFs) and in identifying mechanistic pathways for molecular catalysis taking place in MOF films. Transport phenomena are often overlooked in the kinetic analysis of catalytic reactions occurring in MOFs, resulting in reporting of disguised kinetic parameters (TOFs, Tafel slopes, rate constant, or possibly reaction orders), due to unaccounted interference from mass or charge trans- port. ${ }^{12}$ Fortunately, there are well-developed reaction-diffusion models from mature tangential fields such as electrochemically mediated catalysis within redox polymer-modified electrodes (with covalently or ionically bound transition metal complexes $)^{61-63}$ or using biocatalytic films, ${ }^{64-66}$ either of which may be relevant for analyzing MOF-based catalysts. This section outlines a steady reaction-diffusion kinetic model applicable to a simple one-electron, one-step catalytic reaction facilitated by a molecular catalyst incorporated in a MOF film, where the catalyst species is responsible for both the reaction with the substrate and shuttling of electrons through the film (Figure 4). ${ }^{67,68}$

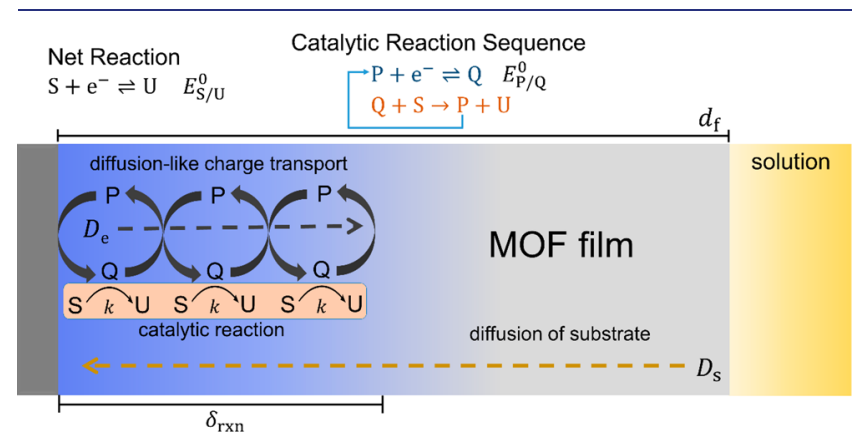

Figure 4. One-electron, one-step catalytic mechanism occurring in a molecular heterogenized MOF film electrode, where $P$ and $Q$ are the oxidized and reduced forms of the catalyst linker, respectively, $\mathrm{S}$ is the substrate, and $k$ is the second-order rate constant for the catalytic reaction $\left(k_{\text {cat }}=k C_{\mathrm{S}}^{0}\right)$.

Primarily three phenomena will determine the overall performance and steady-state current response of any catalytic process taking place within an electroactive MOF:

(1) the intrinsic catalytic reaction

(2) permeation and diffusion of the substrate

(3) diffusional transport of electrons

Additional considerations for electron transfer kinetics at the electrode-film interface or a finite rate of mass transport of the substrate across the film-solution interface appear as boundary conditions in the overall reaction-diffusion problem and have been treated previously. ${ }^{68-71}$ If the substrate is depleted by the reaction and its concentration at the film-solution interface drops below its bulk value, ensuing mass transfer outside the film in solution can be treated as described previously for either rotating disk electrode voltammetry (RDEV) or $\mathrm{CV} .^{63,68}$ However, for simplicity, here we consider the concentration of substrate outside the film to be constant and equal to its bulk value. In general, Fick's laws hold for statistically homogeneous microporous materials, and a continuum model for diffusion and any coupled reactions is valid. ${ }^{14}$ This will certainly apply to MOFs as well; however, as for any microporous material, extensions or adjustments to Fickian diffusion may be considered if required by the particular experimental system at hand. ${ }^{13,14,29,30,72}$ The following discussion focuses on the two diffusional phenomena and the catalytic reaction mentioned above, since these give rise to relativity unfamiliar complications resulting from reaction-diffusion-mainly that the concentration of each species will have a spatial dependence within the film. This is a fundamental characteristic of reactions coupled to diffusion occurring in porous media, where the possible existence of boundary layers, outside of which concentrations 

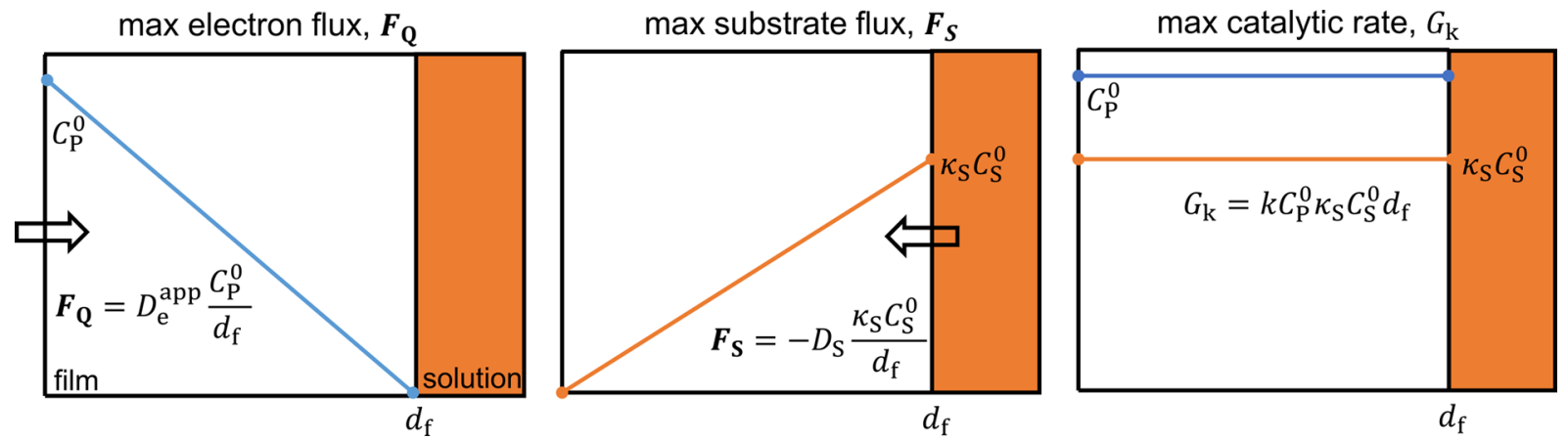

Figure 5. Schematic illustration of limiting fluxes: $F_{\mathrm{Q}}$ for diffusion-like charge transport and $F_{\mathrm{S}}$ for substrate diffusion. ${ }^{78}$ The catalytic reaction is represented by a maximum production or generation rate of product per unit surface area $\left(\mathrm{mol} \mathrm{cm}^{-2} \mathrm{~s}^{-1}\right), G_{\mathrm{k}}$. Concentration profiles for each process are displayed, which give the corresponding characteristic current densities $\left(i_{\mathrm{e}}, i_{\mathrm{s}}\right.$, and $i_{\mathrm{k}}$, respectively) from multiplication of the magnitude of each flux or rate by $F$.

\section{R: catalytic reaction | S: substrate diffusion | E: diffusional charge transport}
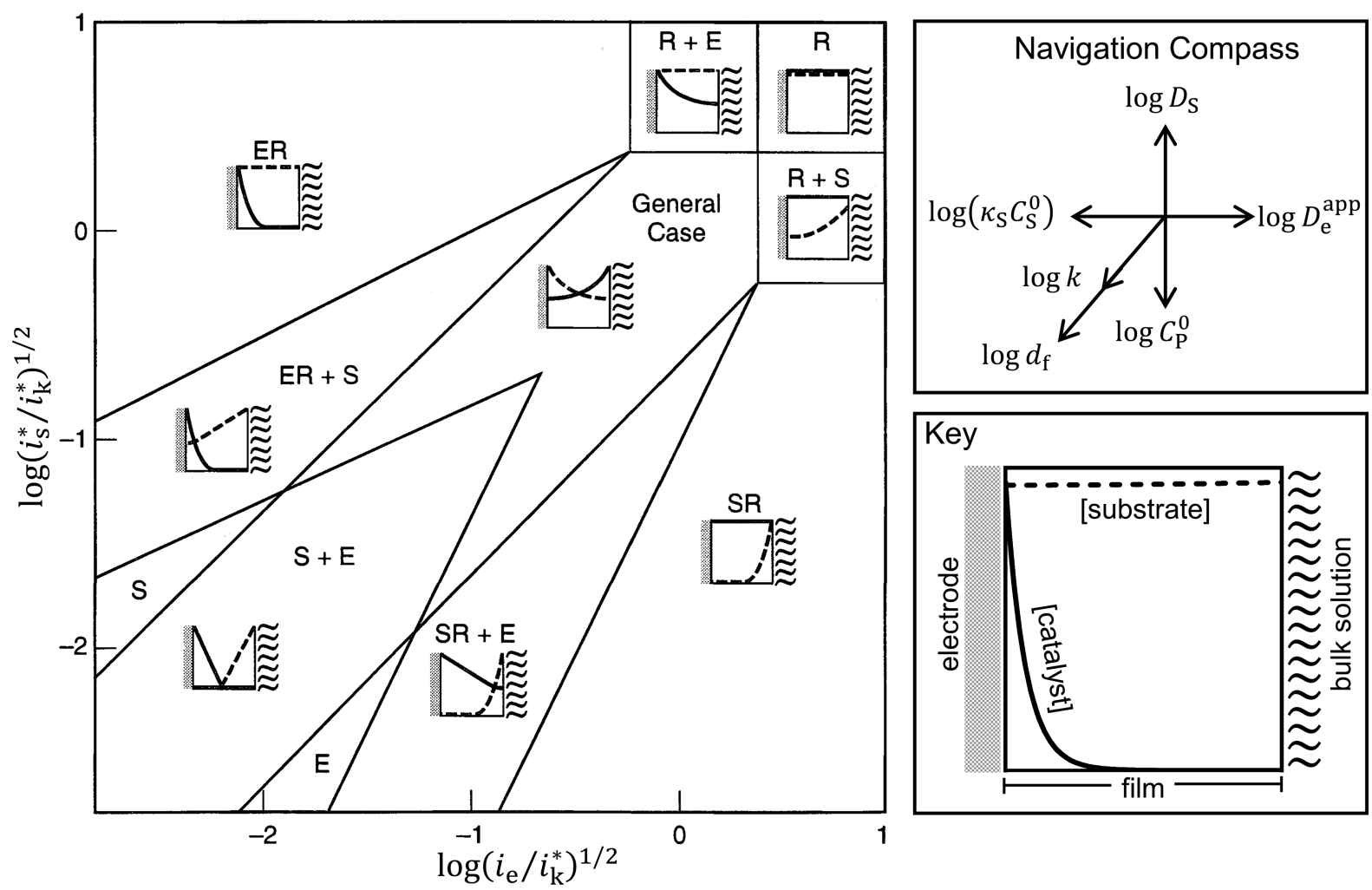

Figure 6. Kinetic zone diagram for limiting cases of a one-step, one-electron catalytic reaction occurring in a multilayer film. ${ }^{69}$ Schematic concentration profiles of reduced catalyst Q (solid line) and substrate $\mathrm{S}$ (dotted line) are displayed in each zone. The definitions of $i_{\mathrm{s}}^{*}$ and $i_{\mathrm{k}}^{*}$ incorporate the possibility for substrate depletion at the film-solution interface and ensuing solution-phase mass transport outside the film. ${ }^{63}$ The navigation compass on the top right shows the magnitude and direction in which each experimental variable will translate the system within the zone diagram. Adapted from ref 69 with permission from Elsevier Science.

of substrate and/or intermediates drop to zero, needs to be taken into account.

Early reports first identified the three primary rate-limiting phenomena, ${ }^{73,74}$ for example by examining the mediated reduction of dibromoalkyl substrates by metalloporphyrin catalysts in multilayer films. ${ }^{75}$ A detailed kinetic model (outlined for steady-state techniques, RDEV) was soon after introduced by Andrieux, Dumas-Bouchiat, and Savéant, ${ }^{61}$ which recently has been updated by Costentin and Savéant for analysis by cyclic voltammetry including the case when the substrate is depleted in solution outside the film (total catalysis). ${ }^{67,68,76}$ The details of this model are briefly recalled in the next section in order to frame its application to molecular catalysis inside MOF films for the analysis that follows.

First of all, a steady-state situation will hold for the dynamical processes in the film when the catalytic reaction is fast as compared to the time scale of the experiment so that there is no accumulation of either reduced catalyst or substrate within the film. ${ }^{68}$ A mass balance on the system in this situation entails that the maximum current will be proportional to either the flux of reduced catalyst at the electrode interface or the flux of substrate at the film-solution interface. ${ }^{64}$ This situation is often referred 

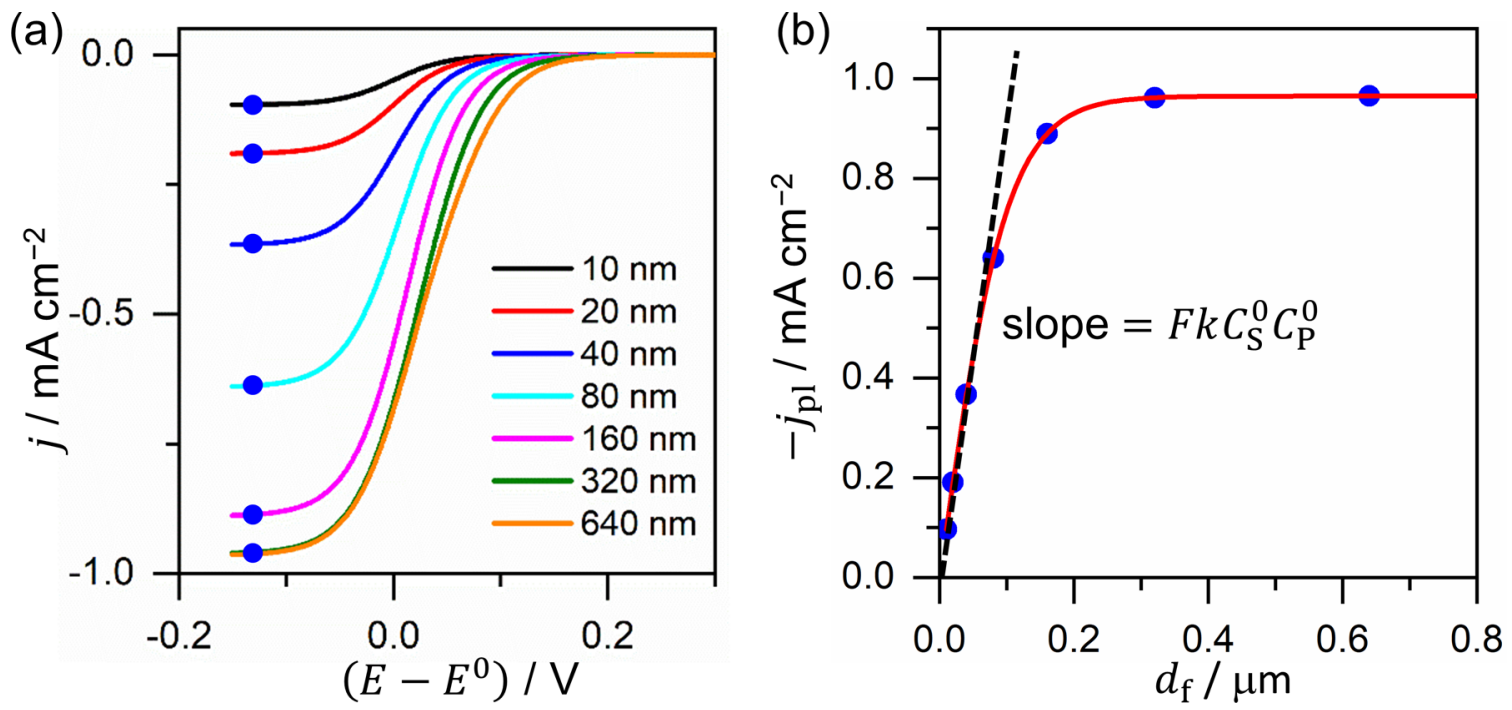

Figure 7. (a) Steady-state CVs with increasing film thickness from $d_{\mathrm{f}}=10 \mathrm{~nm}$ (black) to $d_{\mathrm{f}}=640 \mathrm{~nm}$ (orange). (b) Corresponding plot of steady-state plateau current density $\left(j_{\mathrm{p}}\right.$, blue dots $)$ vs $d_{\mathrm{f}}$ when $i_{\mathrm{s}} \ll i_{\mathrm{e}}$ with $D_{\mathrm{e}}^{\mathrm{app}}=1 \times 10^{-5} \mathrm{~cm}^{2} \mathrm{~s}^{-1}, D_{\mathrm{S}}=1 \times 10^{-10} \mathrm{~cm}^{2} \mathrm{~s}^{-1}, k=10 \mathrm{M}^{-1} \mathrm{~s}^{-1}, C_{\mathrm{S}}^{0}=1 \mathrm{M}, C_{\mathrm{P}}^{0}=0.1 \mathrm{M}$, and $\kappa_{\mathrm{S}}=1$.

to as pure kinetic conditions ${ }^{68,77}$ and becomes important when analyzing reaction-diffusion phenomena of this type, as it simplifies the problem to a form where an analytical or semianalytical solution for the current-potential response may be obtained. The rate of change of any species undergoing reactiondiffusion can be expressed as Fick's second law plus a kinetic term accounting for the coupled chemical reaction. At steady state the time derivative is zero, giving

$$
\begin{aligned}
& D_{\mathrm{e}}^{\mathrm{app}} \frac{\partial^{2} C_{\mathrm{Q}}}{\partial x^{2}}=k C_{\mathrm{Q}} C_{\mathrm{S}} \\
& D_{\mathrm{S}} \frac{\partial^{2} C_{\mathrm{S}}}{\partial x^{2}}=k C_{\mathrm{Q}} C_{\mathrm{S}}
\end{aligned}
$$

where $x$ is the distance normal to the electrode surface with $x=0$ located at the electrode-film interface, and $k$ is the second-order rate constant of the catalytic reaction (see Figure 4). A simultaneous solution to these two equations given the appropriate boundary conditions will render the currentpotential response. ${ }^{61,67,68,76}$

The three rate-limiting factors can each be expressed simply as a characteristic current density: ${ }^{62} i_{\mathrm{k}}, i_{\mathrm{e}}$, and $i_{\mathrm{s}}$, for the catalytic reaction, charge transport, and substrate diffusion, respectively.

$$
\begin{aligned}
& i_{\mathrm{k}}=F k C_{\mathrm{P}}^{0} \kappa_{\mathrm{S}} C_{\mathrm{S}}^{0} d_{\mathrm{f}} \\
& i_{\mathrm{e}}=\frac{F C_{\mathrm{P}}^{0} D_{\mathrm{e}}^{\mathrm{app}}}{d_{\mathrm{f}}} \\
& i_{\mathrm{s}}=\frac{F \kappa_{\mathrm{S}} C_{\mathrm{S}}^{0} D_{\mathrm{s}}}{d_{\mathrm{f}}}
\end{aligned}
$$

where $\kappa_{\mathrm{S}}$ is the equilibrium partition coefficient for substrate crossing the film-solution interface, $C_{\mathrm{P}}^{0}$ is the concentration of redox-active linkers, $C_{S}^{0}$ is the bulk concentration of substrate, and $d_{\mathrm{f}}$ is the film thickness (other terms are defined the same as above). The characteristic current densities are obtained from the hypothetical maximum flux for each potentially limiting process, displayed in Figure 5, and these describe the overall capacity of the film to deliver charge and substrate to the active sites to carry out the catalytic reaction at a given rate. ${ }^{78}$ They represent how the rate of each process (reaction or diffusion) scales under a given set of conditions and independent variables (eq 6). A single process or possibly two processes in parallel, reaction on one hand and a transport phenomenon on the other, may determine the overall observed current.

Akin to Thiele's prediction, the behavior is governed by the ratio of the catalytic rate to either the rate of electron diffusion or substrate diffusion, precisely given by ratios of the characteristic current densities. Therefore, two dimensionless control parameters can be defined, ${ }^{63}$ noting their fundamental similarity to the Thiele modulus in eq 1 :

$$
\begin{aligned}
\sqrt{\frac{i_{\mathrm{k}}}{i_{\mathrm{s}}}} & =d_{\mathrm{f}} \sqrt{\frac{k C_{\mathrm{P}}^{0}}{D_{\mathrm{S}}}} \\
\sqrt{\frac{i_{\mathrm{k}}}{i_{\mathrm{e}}}} & =d_{\mathrm{f}} \sqrt{\frac{k \kappa_{\mathrm{s}} C_{\mathrm{S}}^{0}}{D_{\mathrm{e}}^{\text {app }}}}
\end{aligned}
$$

When one or both parameters are very large, transport limitations by substrate or electron-hopping diffusion are observed along with the appearance of boundary layers, which reduce the total amount of active catalyst within the film under operating conditions. Analytical solutions can be found in many limiting situations when there exists a linear gradient for either $Q$ or $\mathrm{S}$ over a significant dimension of the film. ${ }^{63,68}$ This results in the limiting regimes shown in the zone diagram in Figure $6{ }^{63,68,69}$ It should be noted that there is no analytical solution for the general case (middle, Figure 6), and numerical methods to compute the solution to eq 4 and eq 5 must be employed. ${ }^{79,80}$ In the original formalism, ${ }^{61}$ a nomenclature for the limiting process(es) was established, and the letters $\mathrm{R}, \mathrm{S}$, and $\mathrm{E}$ are assigned to the catalytic reaction, substrate diffusion, and diffusional charge transport being limiting, respectively. Combinations thereof represent mixed kinetic control by two or more phenomena.

Using Film Thickness as a Diagnostic Tool for Reaction-Diffusion. The main analytic tool to diagnose the kinetic limitation occurring within the film is variation of the film thickness. ${ }^{67,68,76}$ This is the parameter that provides the most information and can in principle be varied systematically over a range of values. Very intuitively it can be expected that thicker films will lead to limitations by mass or charge transport. For a 
simple example, consider the case where electron-hopping diffusion is relatively fast $\left(i_{\mathrm{S}} \ll i_{\mathrm{e}}\right)$ compared to the rate at which substrate diffuses through the film, and $\kappa_{\mathrm{S}}$ is approximately unity (Figure 7 ). ${ }^{76}$

Starting with very thin films, the measured catalytic plateau current will be proportional to the film thickness, as the catalytic reaction encompasses the entirety of the volume contained inside the film (zone $\mathrm{R}$, eq 8). With thicker films, this current reaches a limiting value and no longer varies with $d_{\mathrm{f}}$ (zone $\mathrm{SR}$, eq 9). Now, the catalytic reaction and substrate diffusion jointly limit the observed current density. Plotting the plateau current density versus the film thickness, will allow for estimating the intrinsic characteristics of the film. The slope in the limit of thin films is proportional to the intrinsic reaction rate constant $k$, where $C_{\mathrm{P}}^{0}$ and $C_{\mathrm{S}}^{0}$ are either known or can be measured. In the region where the current is invariant with $d_{f}$, the limiting current can be used to calculate $D_{\mathrm{S}}$ (eq 9), since $k$ has been determined. $^{68,76}$

$$
\begin{aligned}
& i_{\mathrm{R}}=F S k C_{\mathrm{P}}^{0} C_{\mathrm{S}}^{0} d_{\mathrm{f}} \\
& i_{\mathrm{SR}}=F S C_{\mathrm{S}}^{0} \sqrt{D_{\mathrm{S}} k C_{\mathrm{P}}^{0}}
\end{aligned}
$$

where $S$ is the geometric surface area $\left(\mathrm{cm}^{2}\right)$. Analogously, when substrate diffusion is much faster than electron hopping $\left(i_{\mathrm{s}} \gg i_{\mathrm{e}}\right)$ and the catalytic reaction is fast compared to diffusion-like charge transport $\left(\sqrt{i_{\mathrm{k}} / i_{\mathrm{e}}} \gg 1\right)$, the steady-state plateau current is given by ${ }^{67}$

$$
i_{\mathrm{ER}}=F S C_{\mathrm{P}}^{0} \sqrt{D_{\mathrm{e}}^{\mathrm{app}} k C_{\mathrm{S}}^{0}}
$$

While the theoretical model presented here is well established, ${ }^{81}$ such an analysis has never, to the best of our knowledge, been done with catalytic MOF films.

Kinetic Analysis of MOF-Based Catalysis Relies on Identifying and Characterizing Boundary Layers. Above, we saw that the current saturates upon increasing the film thickness, perturbing the system into a regime where both reaction and diffusion are now both limiting the overall current. This situation arises principally because the reaction consumes the substrate before it diffuses into the interior of the film forming a boundary layer near the film-solution interface. This is important because the current density in zone SR (eq 9) is proportional to the thickness of the resulting boundary layer. As this becomes thinner, there are fewer active catalysts, and thus, lower overall catalytic efficiency. A scaling relationship of Fick's second law can show how to (very simply) calculate reactiondiffusion layer thickness and total active catalyst concentration. Taking eq 5 when $i_{\mathrm{s}} \ll i_{\mathrm{e}}$ and $\sqrt{i_{\mathrm{k}} / i_{\mathrm{s}}} \gg 1$ (zone SR) and again for simplicity assuming $\kappa_{\mathrm{S}}=1$,

$$
D_{\mathrm{S}} \frac{\partial^{2} C_{\mathrm{S}}}{\partial x^{2}}=k C_{\mathrm{P}}^{0} C_{\mathrm{S}}
$$

If we assume concentrations scale with the corresponding total or bulk concentration of each species (at $E \ll E_{\mathrm{P} / \mathrm{Q}}^{0}$ ) and the distance perpendicular to the electrode surface is rescaled to the size of the reaction-diffusion layer $\left(\delta_{\mathrm{rxn}}\right)$, then eq 11 becomes

$$
D_{\mathrm{S}} \frac{C_{\mathrm{S}}^{0}}{\left(\delta_{\mathrm{rxn}}\right)^{2}} \sim C_{\mathrm{P}}^{0} k C_{\mathrm{S}}^{0}
$$

After rearranging, the reaction-diffusion layer thickness is given by

$$
\delta_{\mathrm{rxn}}=\sqrt{\frac{D_{\mathrm{S}}}{k C_{\mathrm{P}}^{0}}}
$$

The active portion of catalyst $\left(m_{\mathbb{Q}}^{\text {active }}\right.$, with units of moles $)$ can then be found by integrating over the reaction-diffusion layer thickness:

$$
\begin{aligned}
& m_{\mathrm{Q}}^{\text {active }}=S \int_{0}^{d_{\mathrm{f}}} C_{\mathrm{Q}}(x) \mathrm{d} x \approx S \int_{0}^{\delta_{\mathrm{rxn}}} C_{\mathrm{Q}}(x) \mathrm{d} x \\
& m_{\mathrm{Q}}^{\text {active }} \approx S C_{\mathrm{P}}^{0} \delta_{\mathrm{rxn}}
\end{aligned}
$$

Finally, this results in

$$
m_{\mathrm{Q}}^{\text {active }} \approx S \sqrt{\frac{D_{\mathrm{S}} C_{\mathrm{P}}^{0}}{k}}
$$

Interestingly, since the reaction is first-order in substrate, in this situation increasing the bulk concentration of substrate outside the film does not in fact help alleviate the transport limitation by substrate diffusion (the active catalyst concentration is independent of $C_{\mathrm{S}}^{0}$ ). The complementary expression for when charge transport is jointly limiting with the catalytic reaction and substrate diffusion is fast $\left(i_{\mathrm{S}} \gg i_{\mathrm{e}}\right.$ and $\sqrt{i_{\mathrm{k}} / i_{\mathrm{e}}} \gg 1$, zone ER) is

$$
m_{\mathrm{Q}}^{\text {active }} \approx S C_{\mathrm{P}}^{0} \sqrt{\frac{D_{\mathrm{e}}^{\mathrm{app}}}{k C_{\mathrm{S}}^{0}}}
$$

In this case increasing the substrate concentration accelerates the reaction, causes the reaction-diffusion layer to decrease in thickness, and reduces the amount of active catalyst-all exacerbating the limitation by diffusional charge transport. It should be noted that these effects apply to the efficiency of the catalytic film in terms of the fraction of catalyst participating in the reaction, and ultimately put a limit on how thick the film can be made without having unused catalytic sites within the film. Since the reaction is first-order in substrate, increasing the bulk substrate concentration will, however, increase the overall current accordingly (see eqs 8, 9, and 10).

Falsified Kinetics and TOFs That Depend on Film Thickness. Since the chemical reactions occurring in MOFs are innately coupled to diffusive charge or mass transport, situations arise where measured kinetic parameters such as rate constants, reaction orders, TOFs, and activation energies do not reflect intrinsic values. Internal transport limitations by mass or charge diffusion will disguise kinetic values, resulting in what is designated from the traditional chemical engineering vernacular as "falsified kinetics". ${ }^{21}$ In a classic example from basic chemical reaction engineering, ${ }^{13,21}$ the apparent or measured reaction order $n^{\prime}$ in substrate $\left(C_{\mathrm{S}}\right)$ can be related to the true reaction order $n$ by

$$
n^{\prime}(\mathrm{SR})=\frac{n+1}{2}
$$

valid for the situation when substrate diffusion and the catalytic reaction are jointly limiting $\left(i_{\mathrm{S}} \ll i_{\mathrm{e}}\right.$ and $\sqrt{i_{\mathrm{k}} / i_{\mathrm{s}}} \gg 1$; zone $\mathrm{SR}$, i.e., when the substrate is present in a thin boundary layer at the film-solution interface, and $\left.\kappa_{\mathrm{S}}=1\right)$. Conversely, in the case where electron diffusion is slow $\left(i_{\mathrm{e}} \ll i_{\mathrm{S}}\right.$ and $\sqrt{i_{\mathrm{k}} / i_{\mathrm{e}}} \gg 1$, zone $\mathrm{ER})$, the apparent reaction order for substrate is given by

$$
n^{\prime}(\mathrm{ER})=\frac{n}{2}
$$


(i.e., when the reduced catalyst is present in a thin boundary layer at the electrode-film interface). ${ }^{67,77}$ Unless transport limitations are identified or the system is pushed to a regime where only the catalytic reaction is limiting and all gradients are eliminated (see zone R, Figure 6), mechanistic conclusions normally sought from uncorrected kinetic measurements will certainly contain artifacts.

Similarly, using the expression defined in eq 14 , recall that TOFs can depend on film thickness, ${ }^{10,82}$

$$
\begin{aligned}
& \mathrm{TOF}_{\text {max }}^{\text {app }}=\frac{m_{\text {product }}}{m_{\mathrm{Q}}^{\text {total }} t}=\frac{1}{d_{\mathrm{f}}} \sqrt{D_{\mathrm{e}}^{\text {app }} k C_{\mathrm{S}}^{0}} \\
& \mathrm{TOF}_{\text {max }}^{\text {true }}=\frac{m_{\text {product }}^{\text {active }}}{m_{\mathrm{Q}}^{\text {a }} t}=k C_{\mathrm{S}}^{0}
\end{aligned}
$$

where $\mathrm{TOF}_{\text {max }}^{\text {true }}$ reflects the intrinsic reaction rate of the catalyst. This example is given for when $i_{\mathrm{S}} \gg i_{\mathrm{e}}$ and $\sqrt{i_{k} / i_{e}} \gg 1$ (zone ER and $\left.\kappa_{\mathrm{S}}=1\right)$. Indeed, the apparent turnover frequency, $\mathrm{TOF}_{\max }^{\mathrm{app}}$ will have different expressions in each limiting regime, and when transport limitations are present, $\mathrm{TOF}_{\max }^{\mathrm{app}}$ will underestimate $\mathrm{TOF}_{\max }^{\text {true }}$. Additionally, comparing different catalytic MOF films using conflicting TOF values may encumber kinetic benchmarking and comparison between such materials since the film thickness is not necessarily a parameter intrinsic to the material (i.e., it can be varied by using different synthetic routes or fabrication techniques). Consequently, given a new catalytic MOF, diagnosing the limiting regime (zone diagram, Figure 6) under operating conditions, identifying and quantifying any possible boundary layers that may arise, and extracting intrinsic parameters of the system $\left(D_{S}, D_{\mathrm{e}}^{\mathrm{app}}, \mathrm{TOF}_{\max }^{\text {true }}\right)$ are important endeavors to advance the field of MOF-based catalysis.

One experimental example for $\mathrm{CO}_{2}$ reduction (Figure 8) ${ }^{83}$ demonstrates the impact of thick films and ensuing mass or charge transport limitations on the observed current density.

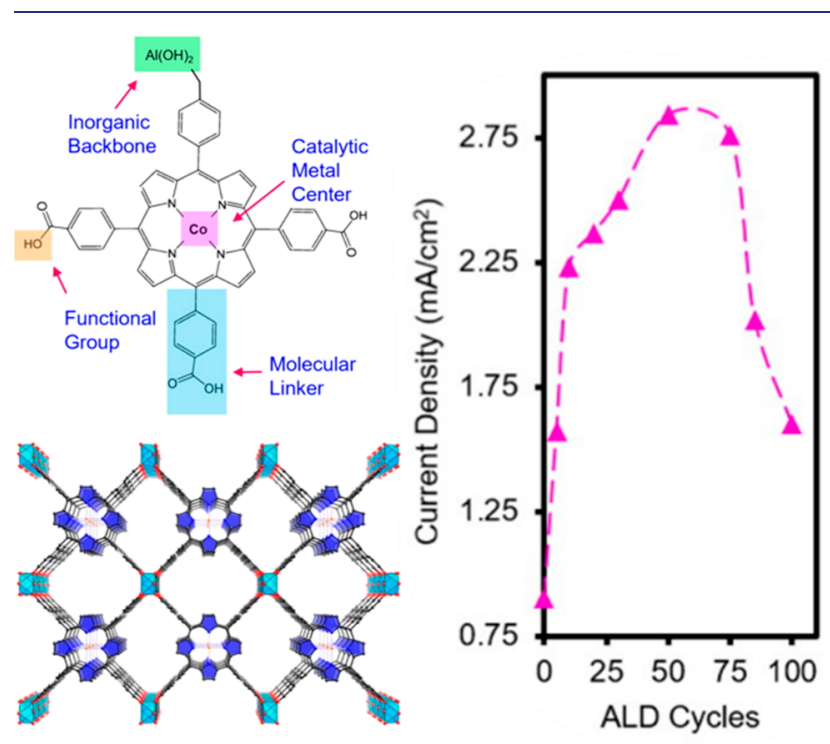

Figure 8. Influence of film thickness on overall steady-state current density demonstrating interference from transport phenomena. $\mathrm{CO}_{2}$ reduction to $\mathrm{CO}$ catalyzed by $\mathrm{Al}_{2}(\mathrm{OH})_{2} \mathrm{TCPP}-\mathrm{Co}\left(\mathrm{TCPP}-\mathrm{H}_{2}=\right.$ $4,4^{\prime}, 4^{\prime \prime}, 4^{\prime \prime \prime}$-(porphyrin-5,10,15,20-tetrayl)tetrabenzoate) films of different thickness (50 ALD cycles correspond to a film thickness approximately of $30-70 \mathrm{~nm}) .{ }^{83}$ Adapted from ref 83 with permission from the American Chemical Society.
Starting from the thinnest films examined, an increase in film thickness led to an approximately linear increase in the total current density (Figure 8), corresponding to the $\mathrm{R}$ limiting regime recalled from the zone diagram in Figure 6. Here the entire film is active since the reaction-diffusion layer is larger than the film thickness (i.e., $\sqrt{i_{\mathrm{k}} / i_{\mathrm{e}}} \ll 1$ or $\sqrt{i_{\mathrm{k}} / i_{\mathrm{S}}} \ll 1$ ), and the transport processes are able to supply charge or substrate across the entire film before being consumed by the reaction. In these limits, turnover frequencies measured in the usual way will yield $\mathrm{TOF}_{\max }^{\text {true }}$. For example, Kornienko et al. reported a TOF value from data taken under these conditions on the order of $0.06 \mathrm{~s}^{-1}$ (Figure 8). ${ }^{83}$

However, upon increasing the film thickness further, the MOF films exhibit a saturation in the total observed current density followed by a sharp decline (Figure 8 ). ${ }^{83}$ The transition from the linear region to this peaked region is expected to correspond to the situation where on the time scale of the reaction, diffusional mass or charge transport are not able to supply electrons or substrate over the entire length of the film (i.e., $\sqrt{i_{\mathrm{k}} / i_{\mathrm{e}}}>1$ or $\left.\sqrt{i_{\mathrm{k}} / i_{\mathrm{S}}}>1\right)$. Since now the film thickness has exceeded the size of the reaction-diffusion layer, a boundary layer would be expected at either the electrode-film interface or the filmsolution interface depending on the relative rates of electronhopping and substrate diffusion (as compared to the catalytic reaction) within the film. Further increase in the film thickness leads to a drop in current as the second diffusional process becomes limiting as well and the film most probably enters either the $\mathrm{SR}+\mathrm{E}$ or $\mathrm{ER}+\mathrm{S}$ situation. ${ }^{68}$ In these limits, as soon as $\sqrt{i_{\mathrm{k}} / i_{\mathrm{e}}}$ or $\sqrt{i_{\mathrm{k}} / i_{\mathrm{S}}}$ becomes greater than unity, $\mathrm{TOF}_{\max }^{\mathrm{app}} \neq \mathrm{TOF}_{\max }^{\text {true }}$ and uncorrected kinetic values will be falsified, since the bulk concentration of catalyst is no longer reflective of the amount of active catalyst contained within the boundary layer. It should be noted, from this example it is not possible to conclude which transport process (either substrate diffusion or charge transport) initially limits the current as the film thickness is increased. This would require additional information, particularly the rate at which the substrate $\left(\mathrm{CO}_{2}\right.$ or possibly protons) diffuse through the MOF films. Additionally, the authors identified several other factors that may contribute to the observed effect on the current density and which may complicate the analysis of these data in terms of reaction-diffusion. For example, $\mathrm{Al}_{2}(\mathrm{OH})_{2}$ TCPP-Co films with the largest thickness may have an underlying layer of aluminum oxide between the conducting FTO surface and the MOF film, which is remaining from the film synthesis and fabrication. ${ }^{83}$ If this layer is sufficiently thick, it would reduce the ohmic contact between the film and conducting substrate, producing lower currents. In short, while this example is one of the few reports to systematically examine the effect of MOF film thickness on catalysis and certainly reveals important effects associated with thick films, overall these results point toward a need for improved methods of film synthesis and fabrication methods for MOFs on electrodes with controllable film thickness and morphology.

\section{OPPORTUNITIES AND CHALLENGES}

Understanding the fundamental kinetics of MOF-based catalysis using molecular active sites is still a major challenge for the field. Where is the bottleneck for catalysis, and how can the unique properties of MOFs be exploited for optimization? Incorporating the foundational principles from the reaction-diffusion models described above, this next section attempts to analyze 

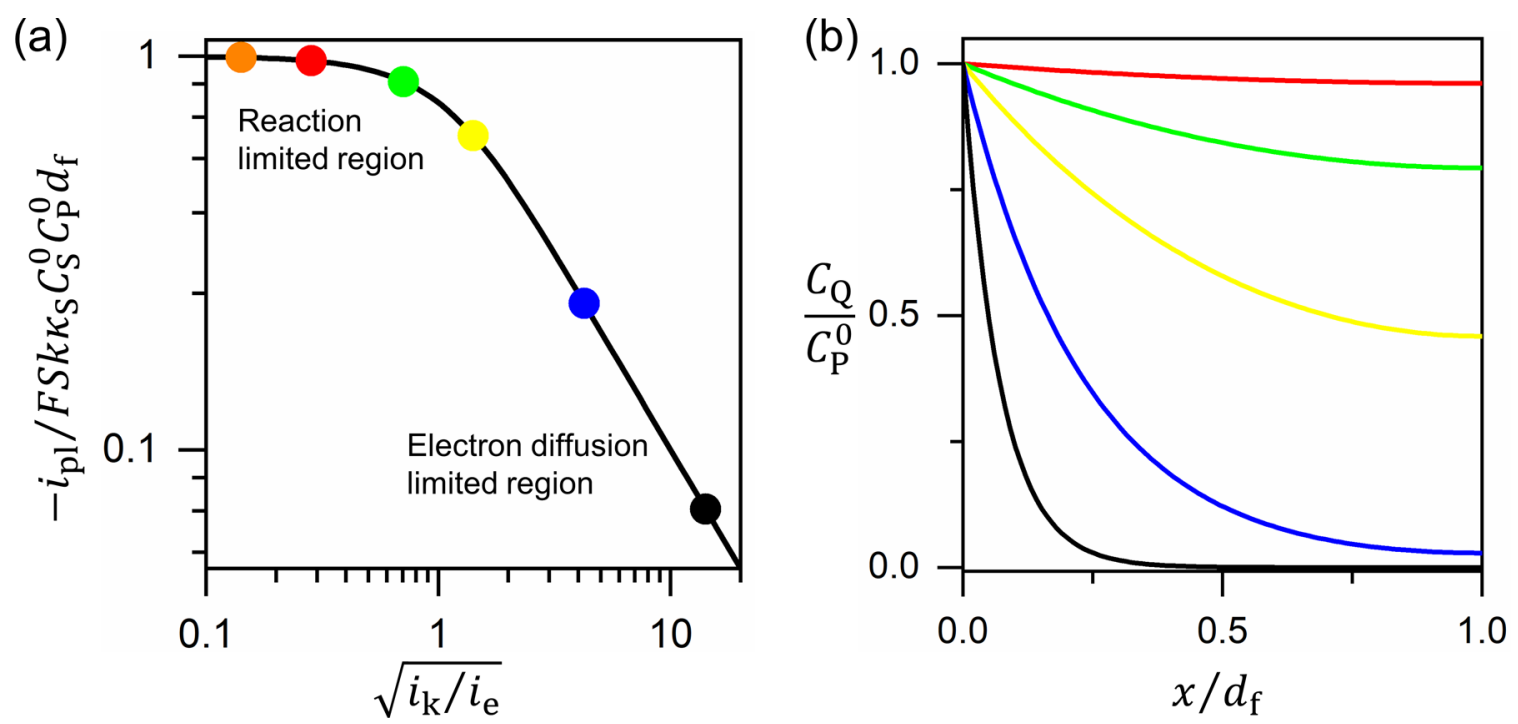

Figure 9. (a) Variation of the steady-state plateau current (at $E \ll E_{\mathrm{P} / \mathrm{Q}}^{0}$ ) with $\sqrt{i_{\mathrm{k}} / i_{\mathrm{e}}}=d_{\mathrm{f}} \sqrt{{k \kappa_{\mathrm{S}}}_{\mathrm{S}} C_{\mathrm{S}}^{0} / D_{\mathrm{e}}^{\mathrm{app}}}$ when substrate concentration is constant throughout the film $\left(i_{\mathrm{S}} \gg i_{\mathrm{e}}\right)$. (b) Corresponding concentration profiles of $\mathrm{Q}: d_{\mathrm{f}}=1 \mu \mathrm{m}, C_{\mathrm{P}}^{0}=1 \mathrm{M}$, and $k \kappa_{\mathrm{S}} C_{\mathrm{S}}^{0}=10 \mathrm{~s}^{-1}$, and $D_{\mathrm{e}}^{\mathrm{app}}=(\mathrm{red}) 1.3 \times 10^{-6} \mathrm{~cm}^{2}$ $\mathrm{s}^{-1}$, (green) $2 \times 10^{-7} \mathrm{~cm}^{2} \mathrm{~s}^{-1}$, (yellow) $5 \times 10^{-8} \mathrm{~cm}^{2} \mathrm{~s}^{-1}$, (blue) $5.5 \times 10^{-9} \mathrm{~cm}^{2} \mathrm{~s}^{-1}$, (black) $5 \times 10^{-10} \mathrm{~cm}^{2} \mathrm{~s}^{-1}$.

each potentially limiting process in turn, with suggestions for increasing catalytic efficiency in ways that are uniquely suited to MOFs.

Limiting Electron Diffusion. As an illustrative example, a situation with limitations by electron-hopping diffusion is displayed in Figure 9. In this situation substrate transport is relatively fast $\left(i_{\mathrm{s}} \gg i_{\mathrm{e}}\right){ }^{67}$ or the bulk concentration of the substrate is very high (for example when the substrate is the solvent). This may be encountered in practice with catalytic MOF films having large pores allowing facile substrate diffusion, but slow charge transport due to a large separation between redox-active linkers ${ }^{28}\left(D_{\mathrm{e}}^{\mathrm{app}}=10^{-10}-10^{-14} \mathrm{~cm}^{2} \mathrm{~s}^{-1}\right){ }^{2}$ Catalytic steady-state current (at $E \ll E_{\mathrm{P} / \mathrm{Q}}^{0}$ ) is plotted versus the dimensionless parameter $\sqrt{i_{\mathrm{k}} / i_{\mathrm{e}}}=d_{\mathrm{f}} \sqrt{k \kappa_{\mathrm{S}} C_{\mathrm{S}}^{0} / D_{\mathrm{e}}^{\text {app }}}$ (Figure 9a), which is the ratio of the catalytic reaction rate to the rate of diffusional charge transport. An insightful alternative definition can be expressed based on a geometric length scale present in the problem $\left(d_{\mathrm{f}}\right)$ and a physical length scale for reaction-diffusion. Consequently, this parameter is also equivalent to the ratio of the film thickness to the thickness of the reaction-diffusion layer. As the electron-hopping diffusion coefficient decreases, the current (normalized to the reaction-limited current, $i_{\mathrm{R}}=$ $\left.F S k \kappa_{\mathrm{S}} C_{\mathrm{S}}^{0} C_{\mathrm{P}}^{0} d_{\mathrm{f}}\right)$ drops markedly. The blue and black dots in Figure 9 and the corresponding concentration profiles in Figure 9 represent electron-hopping diffusion coefficients in a realistic range for electroactive MOF films. ${ }^{30,34,51,84}$ The current in this situation is controlled by both the catalytic reaction and electron diffusion and is no longer a function of film thickness, and the reaction is fast enough compared with diffusion to cause finite gradients of the reduced catalyst to form within the film. Thus, the reaction-diffusion layer is significantly smaller than the film thickness; and therefore, increasing the number of layers (increasing the thickness) is unproductive, because the outer region of the film will not participate in the catalytic reaction. ${ }^{67}$ The $y$-axis in Figure 9 is a measure of catalytic efficiency (how much of the film is active in the reaction), and accordingly it can be seen that large values of $\sqrt{i_{\mathrm{k}} / i_{\mathrm{e}}}$ cause a reaction-diffusion layer to form that reduces the efficiency of the system (i.e., diffusion is not able to support charge transport to all the catalytic sites on the time scale of the reaction, see concentration profiles in Figure 9b).

Limiting Substrate Diffusion. The situation of kinetically limiting substrate diffusion within the film in addition to charge transport is shown in Figure 10. ${ }^{68}$ Representative conditions found for MOF films ${ }^{34,54}$ were used to generate concentration profiles: $D_{\mathrm{e}}^{\mathrm{app}}=5 \times 10^{-10} \mathrm{~cm}^{2} \mathrm{~s}^{-1}, d_{\mathrm{f}}=1 \mu \mathrm{m}, C_{\mathrm{P}}^{o}=1 \mathrm{M}$, with various values of $D_{S}$. An important situation arises when $\sqrt{i_{\mathrm{k}} / i_{\mathrm{s}}}$ $\gg 1$ and $\sqrt{i_{\mathrm{k}} / i_{\mathrm{e}}} \gg 1$. The observed current density is independent of the rate of the catalytic reaction and the only limitations are from substrate diffusion and/or charge transport. This is represented in Figure 10c, displaying zone S+E. In fact, when the transport processes are much slower than the reaction rate, the reaction-diffusion layer approaches the size of a monolayer, which can be located at either interface (either the electrode-film interface, zone $S$, or the film-solution interface, zone E). ${ }^{61,64}$ The overall current density is plotted in Figure 10e for each value of $D_{S}$, corresponding to the concentration profiles in Figure $10 \mathrm{a}-\mathrm{d}$. Typical surface concentrations and electronhopping transport parameters reported in literature indeed show that catalytic MOF films are able to reach the recognized minimum $10 \mathrm{~mA} \mathrm{~cm}^{-2}$ needed for large-scale applications ${ }^{85}$ with a moderately active catalyst (25 turnovers per second); however, with a typical film thicknesses $d_{\mathrm{f}}=1 \mu \mathrm{m}$ the efficiency is rather low (see the shaded region in Figure 10 indicating the size of the reaction-diffusion layer). Importantly, this assumes facile substrate transport through the film (Figure 10, $D_{\mathrm{S}}=10^{-5} \mathrm{~cm}^{2}$ $\mathrm{s}^{-1}$ ) more typical of a freely diffusing species in solution. As the substrate diffusion coefficient is decreased below $10^{-7} \mathrm{~cm}^{2} \mathrm{~s}^{-1}$ while holding all other values constant, a sharp decline in steadystate current density is observed (Figure 10e). As an example of typical diffusion rates, the diffusivities of tertiary amines (triethylamine, tripropylamine, etc.) through solvent-filled pores of a $\mathrm{Zn} \mathrm{MOF}\left(\left[\mathrm{Zn}_{2} \mathrm{~L}_{0.5}(\mathrm{BPDC})_{2}\right]_{3} \cdot 9 \mathrm{DMF} \cdot 9 \mathrm{H}_{2} \mathrm{O}\right)$ were quantified in the range of $10^{-9}-10^{-10} \mathrm{~cm}^{2} \mathrm{~s}^{-1}$. ${ }^{86}$ However, we note that alkyl amines may not be representative of typical small molecule substrates for energy conversion applications. Clearly there is room for the field and in particular for electrochemistry 

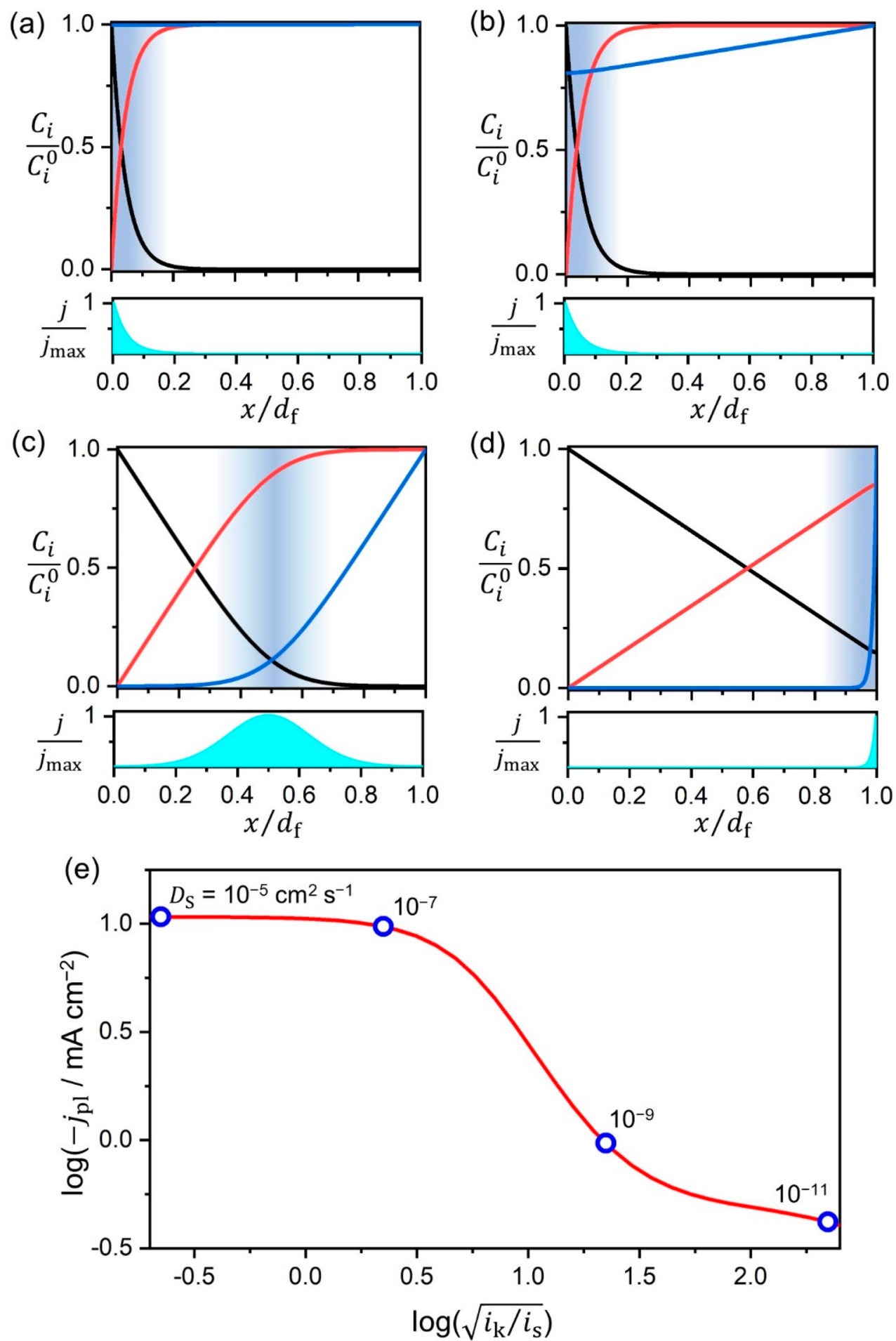

Figure 10. Progressively slower substrate diffusion: simulated concentration profiles of oxidized catalyst $P$ (red), reduced catalyst $Q$ (black), and substrate $S$ (blue) using finite difference method using $D_{\mathrm{e}}^{\mathrm{app}}=5 \times 10^{-10} \mathrm{~cm}^{2} \mathrm{~s}^{-1}, d_{\mathrm{f}}=1 \mu \mathrm{m}, C_{\mathrm{P}}^{0}=1 \mathrm{M}, \kappa_{\mathrm{S}} C_{\mathrm{S}}^{0}=0.1 \mathrm{M}$, and $k \kappa_{\mathrm{S}} C_{\mathrm{S}}^{0}=25 \mathrm{~s}^{-1}$, with $D_{\mathrm{S}}$ from $(\mathrm{a})$ $5 \times 10^{-5}$, (b) $5 \times 10^{-7}$, (c) $5 \times 10^{-9}$, to (d) $5 \times 10^{-11} \mathrm{~cm}^{2} \mathrm{~s}^{-1}$. Substrate concentration within the film (blue lines, $C_{\mathrm{S}}$ ) is normalized to the substrate concentration at the film-solution interface (equal to $\kappa_{\mathrm{S}} C_{S}^{0}$ ). Below each concentration profile the normalized local current density (cyan) as a function of distance from the electrode $(x)$ normalized to the film thickness is shown. The shaded region indicates the location of the reaction-diffusion layer inside the film. (e) Calculated steady-state current density (at $E \ll E_{\mathrm{P} / \mathrm{Q}}^{0}$ ) corresponding to the concentration profiles in part (a) through (d) plotted as a function of $\sqrt{i_{\mathrm{k}} / i_{\mathrm{S}}}$ with the value of $D_{\mathrm{S}}$ shown next to each point. Other parameters are the same as above $\left(D_{\mathrm{e}}^{\mathrm{app}}=5 \times 10^{-10} \mathrm{~cm}^{2} \mathrm{~s}^{-1}, d_{\mathrm{f}}=1 \mu \mathrm{m}, C_{\mathrm{P}}^{0}=1\right.$ $\mathrm{M}, \kappa_{\mathrm{S}} C_{\mathrm{S}}^{0}=0.1 \mathrm{M}$, and $k \kappa_{\mathrm{S}} C_{\mathrm{S}}^{0}=25 \mathrm{~s}^{-1}$ ).

as a technique to aid in the quantitative measurement of diffusion rates of small molecules through MOFs under conditions relevant to catalysis (solvated). Overall, the simulated results presented here preliminarily suggest that a combination of substrate diffusion and charge transport may be kinetically limiting in many MOF-based catalytic films of 

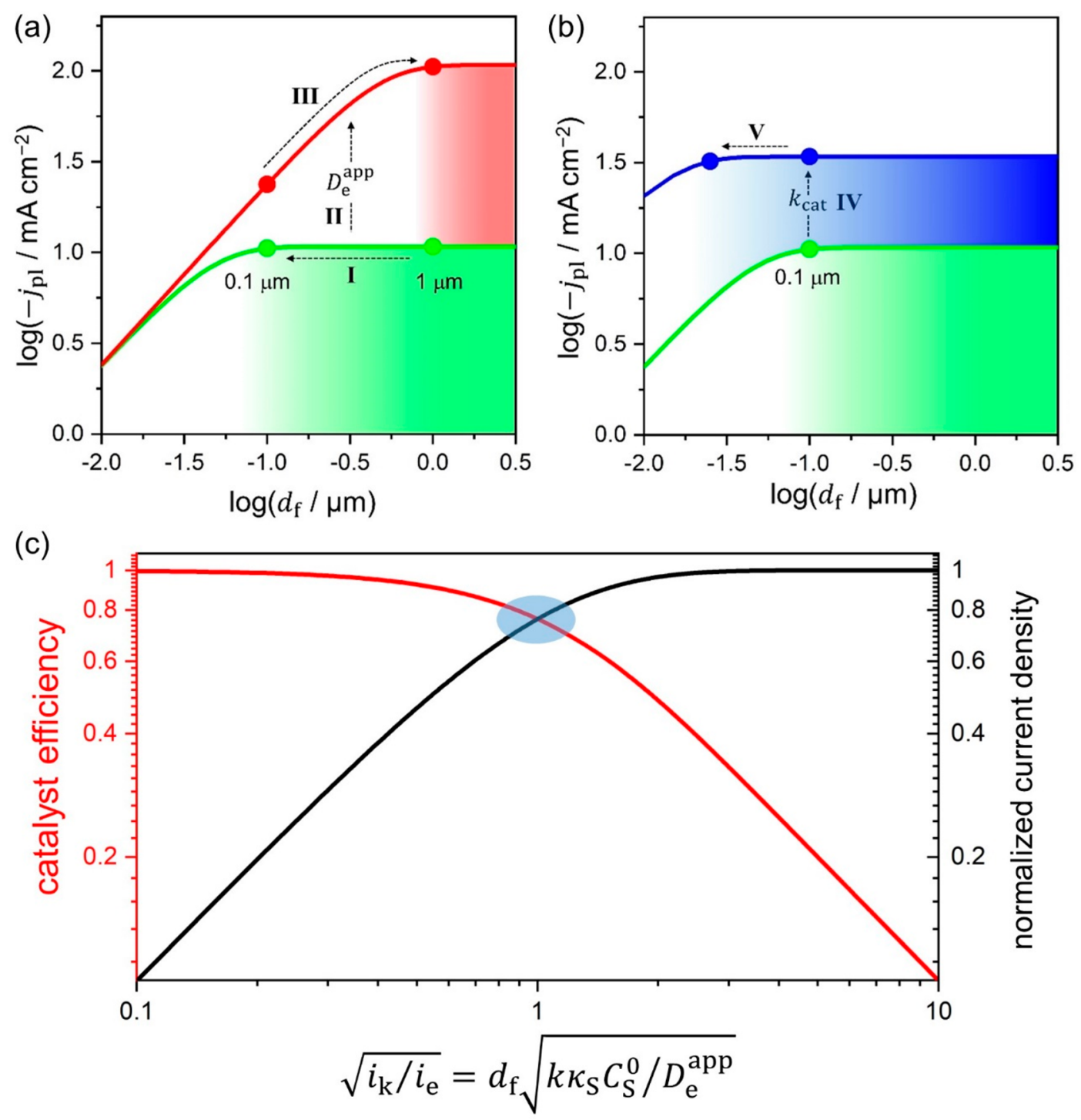

Figure 11. (a) Options for optimizing MOF-based molecular catalysis either by adjustment of the film thickness or by increasing one or more diffusion coefficients by modifying the pore structure/properties via linker design. The example shown here applies to when electron-hopping diffusion is much slower than substrate transport $\left(i_{\mathrm{s}} \gg i_{\mathrm{e}}\right)$. The shaded region below each curve signifies film thicknesses where the efficiency is less than unity and the amount of active catalyst is less than the amount of total catalyst. In (a), the observed current density is plotted versus the film thickness $\left(d_{\mathrm{f}}\right)$ for two different values of $D_{\mathrm{e}}^{\mathrm{app}}, 5 \times 10^{-10} \mathrm{~cm}^{2} \mathrm{~s}^{-1}$ (green curve) and $5 \times 10^{-8} \mathrm{~cm}^{2} \mathrm{~s}^{-1}$ (red curve). Other parameters are the same as above: $C_{\mathrm{P}}^{0}=1 \mathrm{M}, \kappa_{\mathrm{S}} C_{\mathrm{S}}^{0}=0.1$ $\mathrm{M}$, and $k \kappa_{\mathrm{S}} C_{\mathrm{S}}^{0}=25 \mathrm{~s}^{-1}$. (b) Effect of different intrinsic catalyst turnover frequencies, $k \kappa_{\mathrm{S}} C_{\mathrm{S}}^{0}=25 \mathrm{~s}^{-1}$ (green curve) and $k \kappa_{\mathrm{S}} C_{\mathrm{S}}^{0}=250 \mathrm{~s}^{-1}$ (blue curve), on observed current density with $D_{\mathrm{e}}^{\mathrm{app}}=5 \times 10^{-10} \mathrm{~cm}^{2} \mathrm{~s}^{-1}, C_{\mathrm{P}}^{0}=1 \mathrm{M}$, and $\kappa_{\mathrm{S}} C_{\mathrm{S}}^{0}=0.1 \mathrm{M}$. (c) Double logarithmic plot of catalyst efficiency (red curve, left axis) and observed current density normalized to the electron diffusion limited current density, $j_{\mathrm{pl}} / F C_{\mathrm{P}}^{0} \sqrt{D_{\mathrm{e}}^{\text {app }} k \kappa_{\mathrm{S}} C_{\mathrm{S}}^{0}}$ (black curve, right axis), versus the dimensionless control parameter $\sqrt{i_{\mathrm{k}} / i_{\mathrm{e}}}=d_{\mathrm{f}} \sqrt{k \kappa_{\mathrm{S}} C_{\mathrm{S}}^{0} / D_{\mathrm{e}}^{\text {app }}}$. Optimal catalytic performance is obtained at the intersection of these two curves when $\sqrt{i_{\mathrm{k}} / i_{\mathrm{e}}}=1$ (shaded circle).

average thickness $(\sim 1 \mu \mathrm{m})$; however, certainly more experimental studies addressing catalysis and transport phenomena are needed to fully assign the kinetic behavior of many catalytic MOFs.

Opportunities. Future efforts toward improving or designing molecular catalysis in MOF films should include utilizing reaction-diffusion models (such as the simple one recalled above) to characterize charge and mass transport rates. However, importantly, deployment of these types of porous catalyst materials at scale requires optimizing both catalyst reactivity and transport properties concurrently. Avoiding transport limitations might require decelerating the catalytic rate so that it is slower than diffusive transport by operating the catalytic MOF film at lower current densities. However, this is not very effective for fast conversion of substrate to product on a large scale. Conversely, running the overall process at higher current densities (where the catalytic reaction is much faster than the diffusional processes) quickly becomes inefficient as transport limitations restrict the amount of active catalyst via the formation of boundary layers. Finding the most efficient operating conditions between these two scenarios can only be done when the intrinsic chemical reactivity of the catalyst toward the substrate and the transport properties of the catalyst support matrix are examined, characterized, and optimized together. For MOF-based molecular catalysis, this means that modifying the reactivity of the molecular catalyst alone is not enough to progress 
toward realistic applications of these materials, and significant efforts must be made to design these systems so as to balance chemical reactivity with the diffusional transport phenomena outlined here.

Once the transport parameters (diffusion coefficients) and intrinsic reaction kinetics are measured and the rate-limiting process or processes is identified, a clear step forward is to improve these limitations by one of two methods. As shown above, MOF films are indeed able to reach high current densities of industrial relevance $\left(10 \mathrm{~mA} \mathrm{~cm}{ }^{-2}\right)$. However, often the efficiency (what fraction of the catalysts are active in the film) is less than ideal, making these systems difficult to scale-up to meet requirements for applications. The first and most straightforward strategy is to modify the film to an optimal thickness where $d_{\mathrm{f}} \sqrt{\frac{k C_{\mathrm{P}}^{0}}{D_{\mathrm{S}}}} \approx 1$ and $d_{\mathrm{f}} \sqrt{\frac{k \kappa_{\mathrm{s}} C_{\mathrm{S}}^{0}}{D_{\mathrm{e}}^{\text {app }}}} \approx 1 .{ }^{15,67,68}$ Practical methods to make MOF films in a controlled manner are critical in this endeavor. ${ }^{87,88}$ Optimizing film thickness is displayed in Figure 11 (green curve) assuming facile substrate transport for simplicity, where starting from $D_{\mathrm{e}}^{\mathrm{app}}=5 \times 10^{-10} \mathrm{~cm}^{2} \mathrm{~s}^{-1}$, a 1 $\mu \mathrm{m}$ film produces $10 \mathrm{~mA} \mathrm{~cm}{ }^{-2}$ of current. Decreasing the film thickness from $1 \mu \mathrm{m}$ to $100 \mathrm{~nm}$ (process I in Figure 11a) improves the overall efficiency to where nearly all the catalysts are active, while maintaining the same current density. However, the rates of substrate and electron-hopping diffusion set an upper limit on how thick the film can be made (i.e., no larger than the reaction-diffusion layer thickness), and thus bounds the overall rate of product formation. Alternatively, tuning the molecular building blocks is a unique property of MOFs that can be used to modify macroscopic transport properties-something that is relatively more difficult to accomplish in other porous materials lacking molecularly discrete components. Using the same example (Figure 11a), increasing $D_{\mathrm{e}}^{\text {app }}$ from 5 $\times 10^{-10} \mathrm{~cm}^{2} \mathrm{~s}^{-1}$ to $5 \times 10^{-8} \mathrm{~cm}^{2} \mathrm{~s}^{-1}$ (process II in Figure 11a) gives an even higher current density of about $20 \mathrm{~mA} \mathrm{~cm}^{-2}$ and unity catalyst efficiency with the same $100 \mathrm{~nm}$ film (Figure 11, red curve). Now with faster charge transport properties, the catalytic MOF film can be made thicker (up to the point where $d_{\mathrm{f}} \sqrt{\frac{k k_{\mathrm{s}} C_{\mathrm{s}}^{o}}{D_{\mathrm{e}}^{\text {app }}}} \approx 1$, process III in Figure 11a) without compromising efficiency, reaching a maximum of $100 \mathrm{~mA} \mathrm{~cm}^{-2}$ with $1 \mu \mathrm{m}$ thick films. Designing the pore environment to facilitate charge or substrate transport by synthetic tuning of the linkers or by wellestablished reticular chemistry ${ }^{89,90}$ is thus a distinct advantage available to MOF materials. Ultimately, this will allow thicker films to be used, which do not display transport limitations, giving higher overall catalyst efficiency and higher rates of production.

It is interesting to note that the current densities in this simple example $\left(\geq 10 \mathrm{~mA} \mathrm{~cm}^{-2}\right)$ could be achieved with a relatively slow intrinsic rate of turnover $\left(25 \mathrm{~s}^{-1}\right)$ for the molecular catalyst covalently incorporated in the MOF. This is certainly due to the relatively high concentration of active sites within the MOF film $\left(10-100 \mathrm{nmol} \mathrm{cm}^{-2}\right.$ is a typical range $\left.\mathrm{e}^{32,34,83}\right)$. If the intrinsic rate of the molecular catalyst were increased further, this would allow one to make thinner films utilizing less material and achieve higher current densities. This is shown in Figure 11 where an increasing in $k_{\text {cat }}$ by an order of magnitude for the same charge transport rate $\left(D_{\mathrm{e}}^{\mathrm{app}}=5 \times 10^{-10} \mathrm{~cm}^{2} \mathrm{~s}^{-1}\right.$ and $\left.d_{\mathrm{f}}=0.1 \mu \mathrm{m}\right)$ increases the observed current density from 10 to $30 \mathrm{~mA} \mathrm{~cm}{ }^{-2}$ (process IV, Figure 11b). Reducing the film thickness (process $\mathrm{V}$, Figure $11 \mathrm{~b}$ ) is then an option for achieving optimal efficiency.
Overall, a common theme in all the examples presented here is the realization that reaction-diffusion leads to a balance between catalyst efficiency (amount of used catalyst within the film) and the total rate of production (observed current density), as depicted in Figure 11c. Optimal catalytic performance (highest efficiency and highest current density) is obtained when $\sqrt{i_{\mathrm{k}} / i_{\mathrm{e}}}=$ 1 (Figure 11c, intersection between red and black curves). ${ }^{67}$

\section{CONCLUSION}

Metal-organic frameworks are excellent candidates for support matrices in which to incorporate molecular catalysts. A major advantage to this strategy is the synthetic tunability of both the framework and the catalyst at the molecular scale. At present, however, a notable barrier to this realization and to the eventual application of these materials to catalytic processes at industrial scale is the coupling of transport phenomena to traditional chemical kinetics, as a consequence of immobilization of the molecular species in a finite 3D porous structure. However, once transport by diffusion is quantitatively analyzed and taken into account using simple and well-established reaction-diffusion kinetic models, first introduced by Thiele and Damköhler in the 1930s, optimizing the pore structure of the framework for facile diffusive transport could yield highly efficient catalytic materials as well as a deeper understanding of the intrinsic kinetics and mechanisms of the molecular catalyst under operating condition inside the MOF-film structure. MOFs are uniquely suited to this task due to the structure-property relationship between their molecular-level building blocks and their macroscopic physical and chemical properties. The kinetic information gained from the analysis presented here can be in turn used to inform molecular-level design that will best elevate metal-organic frameworks toward becoming suitable for applications as highperformance catalysts for the electrochemical activation and conversion of small molecules.

\section{ASSOCIATED CONTENT}

\section{Supporting Information}

The Supporting Information is available free of charge at https://pubs.acs.org/doi/10.1021/jacs.0c02899.

$\mathrm{CV}$ simulations and corresponding concentration profiles of electron-hopping diffusion occurring in a MOF film (MP4)

\section{AUTHOR INFORMATION}

\section{Corresponding Author}

Sascha Ott - Department of Chemistry - Ångström Laboratory, Uppsala University, 75120 Uppsala, Sweden; ำ orcid.org/ 0000-0002-1691-729X; Email: sascha.ott@kemi.uu.se

\section{Authors}

Ben A. Johnson - Department of Chemistry - Angström Laboratory, Uppsala University, 75120 Uppsala, Sweden; (1) orcid.org/0000-0002-6570-6392

Anna M. Beiler - Department of Chemistry - Ångstrom Laboratory, Uppsala University, 75120 Uppsala, Sweden

Brian D. McCarthy - Department of Chemistry - Angström Laboratory, Uppsala University, 75120 Uppsala, Sweden; (1) orcid.org/0000-0002-2792-1681

Complete contact information is available at:

https://pubs.acs.org/10.1021/jacs.0c02899 


\section{Notes}

The authors declare no competing financial interest.

\section{ACKNOWLEDGMENTS}

Financial support from the European Research Council via Project ERC-CoG2015-681895_MOFcat is gratefully acknowledged. A.M.B. was supported by the Olle Engkvist Byggmästare Foundation.

\section{SYMBOLS}

$C_{j}$
$C_{\mathrm{P}}^{o}$

concentration of species $j\left(\mathrm{M}, \mathrm{mol} \mathrm{cm}{ }^{-3}\right)$

$\mathrm{C}_{\mathrm{P}}^{o} \quad$ total electroactive concentration of redox-active linker, $\mathrm{P}\left(\mathrm{M}, \mathrm{mol} \mathrm{cm}{ }^{-3}\right)$

$\mathrm{C}_{\mathrm{S}}^{o} \quad$ bulk concentration of substrate $\left(\mathrm{M}, \mathrm{mol} \mathrm{cm}{ }^{-3}\right)$

$d_{\mathrm{f}} \quad$ film thickness $(\mathrm{cm})$

$d \quad$ average hopping distance $(\mathrm{cm})$

$D_{\mathrm{e}} \quad$ electron hopping diffusion coefficient $\left(\mathrm{cm}^{2} \mathrm{~s}^{-1}\right)$

$D_{\mathrm{e}}^{\mathrm{app}} \quad$ apparent charge transport diffusion coefficient $\left(\mathrm{cm}^{2}\right.$ $\left.\mathrm{s}^{-1}\right)$

$D_{\mathrm{S}} \quad$ intra-MOF diffusion coefficient of substrate $\left(\mathrm{cm}^{2} \mathrm{~s}^{-1}\right)$

E electrode potential (V)

$E^{0} \quad$ standard potential (V)

$F_{j} \quad$ molar flux of species $j\left(\mathrm{~mol} \mathrm{~cm}^{-2} \mathrm{~s}^{-1}\right)$

$F \quad$ Faraday constant $\left(\mathrm{C} \mathrm{mol}^{-1}\right)$

$G_{\mathrm{k}} \quad$ production/consumption rate of chemical reaction per unit surface area $\left(\mathrm{mol} \mathrm{cm} \mathrm{cm}^{-2}\right)$

$i \quad$ current (A)

$i_{\mathrm{pl}} \quad$ plateau or limiting steady state current (A)

$i_{\text {s }} \quad$ characteristic current density for substrate diffusion within film $\left(\mathrm{A} \mathrm{cm}^{-2}\right)$

$i_{\mathrm{e}} \quad$ characteristic current density for diffusional charge transport $\left(\mathrm{A} \mathrm{cm}^{-2}\right)$

$i_{\mathrm{k}} \quad$ characteristic current density for catalytic reaction (A $\mathrm{cm}^{-2}$ )

j current density $\left(\mathrm{A} \mathrm{cm}^{-2}\right)$

$j_{\mathrm{pl}} \quad$ plateau or limiting steady state current density (A $\mathrm{cm}^{-2}$ )

$k \quad$ second order rate constant of chemical step $\left(\mathrm{M}^{-1} \mathrm{~s}^{-1}\right.$, $\mathrm{cm}^{3} \mathrm{~mol}^{-1} \mathrm{~s}^{-1}$ )

$k_{\text {cat }} \quad$ observed first order catalytic rate constant $\left(\mathrm{s}^{-1}\right)$

$k_{e} \quad$ self-exchange rate constant occurring only in direction normal to electrode $\left(\mathrm{M}^{-1} \mathrm{~s}^{-1}, \mathrm{~cm}^{3} \mathrm{~mol}^{-1} \mathrm{~s}^{-1}\right)$

$k_{\text {ex }} \quad$ self-exchange rate constant $\left(\mathrm{M}^{-1} \mathrm{~s}^{-1}, \mathrm{~cm}^{3} \mathrm{~mol}^{-1} \mathrm{~s}^{-1}\right)$

$k_{\mathrm{s}} \quad$ standard interfacial rate constant $\left(\mathrm{cm} \mathrm{s}^{-1}\right)$

$m_{\mathrm{Q}}^{\text {active }}$ moles of active catalyst in MOF film (mol)

$n^{\prime}$ apparent reaction order in substrate

true reaction order in substrate

radial distance $(\mathrm{cm})$

$\begin{array}{ll}R & \text { gas constant }\left(\mathrm{J} \mathrm{mol}^{-1} \mathrm{~K}^{-1}\right) \\ R_{2} & \text { MOF particle radius }(\mathrm{cm})\end{array}$

$S \quad$ surface area $\left(\mathrm{cm}^{2}\right)$

$t \quad$ time (s)

TOF $_{\max }^{\text {app }}$ maximum apparent turnover frequency $\left(\mathrm{s}^{-1}\right)$

TOF $_{\max }^{\text {true }}$ maximum true turnover frequency $\left(\mathrm{s}^{-1}\right)$

$x$ distance in Cartesian coordinates perpendicular to electrode surface $(\mathrm{cm})$

$\delta_{\mathrm{rxn}} \quad$ reaction-diffusion layer thickness $(\mathrm{cm})$

$\delta \quad$ diffusion layer thickness $(\mathrm{cm})$

$\kappa_{\mathrm{S}} \quad$ partition equilibrium constant for substrate crossing MOF film-solution interface

$\nu \quad$ scan rate $\left(\mathrm{V} \mathrm{s}^{-1}\right)$

\section{REFERENCES}

(1) Downes, C. A.; Marinescu, S. C. Electrocatalytic Metal-Organic Frameworks for Energy Applications. ChemSusChem 2017, 10 (22), 4374-4392.

(2) McCarthy, B. D.; Beiler, A. M.; Johnson, B. A.; Liseev, T.; Castner, A. T.; Ott, S. Analysis of Electrocatalytic Metal-Organic Frameworks. Coord. Chem. Rev. 2020, 406, 213137.

(3) Majewski, M. B.; Peters, A. W.; Wasielewski, M. R.; Hupp, J. T.; Farha, O. K. Metal-Organic Frameworks as Platform Materials for Solar Fuels Catalysis. ACS Energy Lett. 2018, 3 (3), 598-611.

(4) Zhou, H.-C.; Long, J. R.; Yaghi, O. M. Introduction to MetalOrganic Frameworks. Chem. Rev. 2012, 112 (2), 673-674.

(5) Furukawa, H.; Cordova, K. E.; O’Keeffe, M.; Yaghi, O. M. The Chemistry and Applications of Metal-Organic Frameworks. Science 2013, 341 (6149), 123044.

(6) Tanabe, K. K.; Cohen, S. M. Postsynthetic Modification of MetalOrganic Frameworks-a Progress Report. Chem. Soc. Rev. 2011, 40 (2), 498-519.

(7) Cohen, S. M. The Postsynthetic Renaissance in Porous Solids. J. Am. Chem. Soc. 2017, 139 (8), 2855-2863.

(8) Cohen, S. M.; Zhang, Z.; Boissonnault, J. A. Toward "MetalloMOFzymes": Metal-Organic Frameworks with Single-Site Metal Catalysts for Small-Molecule Transformations. Inorg. Chem. 2016, 55 (15), 7281-7290.

(9) Farha, O. K.; Eryazici, I.; Jeong, N. C.; Hauser, B. G.; Wilmer, C. E.; Sarjeant, A. A.; Snurr, R. Q.; Nguyen, S. T.; Yazaydın, A. Ö.; Hupp, J. T. Metal-Organic Framework Materials with Ultrahigh Surface Areas: Is the Sky the Limit? J. Am. Chem. Soc. 2012, 134 (36), 15016-15021.

(10) Costentin, C.; Savéant, J.-M. Molecular Approach to Catalysis of Electrochemical Reaction in Porous Films. Curr. Opin. Electrochem. 2019, 15, 58-65.

(11) Yang, D.; Gaggioli, C. A.; Ray, D.; Babucci, M.; Gagliardi, L.; Gates, B. C. Tuning Catalytic Sites on Zr6O8Metal-Organic Framework Nodes via Ligand and Defect Chemistry Probed with Tert-Butyl Alcohol Dehydration to Isobutylene. J. Am. Chem. Soc. 2020, $142,8044$.

(12) Yang, D.; Gates, B. C. Catalysis by Metal Organic Frameworks: Perspective and Suggestions for Future Research. ACS Catal. 2019, 9 (3), 1779-1798.

(13) Kärger, J.; Ruthven, D. M.; Theodorou, D. N. Diffusion in Nanoporous Materials; Wiley-VCH Verlag GmbH \& Co. KGaA, 2012.

(14) Kärger, J.; Ruthven, D. M. Diffusion in Nanoporous Materials: Fundamental Principles. New J. Chem. 2016, 40 (5), 4027-4048.

(15) Bediako, D. K.; Costentin, C.; Jones, E. C.; Nocera, D. G.; Savéant, J.-M. Proton-Electron Transport and Transfer in Electrocatalytic Films. Application to a Cobalt-Based O 2 -Evolution Catalyst. J. Am. Chem. Soc. 2013, 135 (28), 10492-10502.

(16) Costentin, C.; Savéant, J.-M. Concepts and Tools for Mechanism and Selectivity Analysis in Synthetic Organic Electrochemistry. Proc. Natl. Acad. Sci. U. S. A. 2019, 116 (23), 11147-11152.

(17) Francke, R.; Little, R. D. Redox Catalysis in Organic Electrosynthesis: Basic Principles and Recent Developments. Chem. Soc. Rev. 2014, 43 (8), 2492-2521.

(18) Wiebe, A.; Gieshoff, T.; Möhle, S.; Rodrigo, E.; Zirbes, M.; Waldvogel, S. R. Electrifying Organic Synthesis. Angew. Chem., Int. Ed. 2018, 57 (20), 5594-5619.

(19) Thiele, E. W. Relation between Catalytic Activity and Size of Particle. Ind. Eng. Chem. 1939, 31 (7), 916-920.

(20) Davis, M. E.; Davis, R. J. Fundamentals of Chemical Reaction Engineering, 1st ed.; McGraw-Hill: New York, 2003.

(21) Fogler, H. S. Elements of Chemical Reaction Engineering, 5th ed.; Pearson Education Inc., 2016.

(22) Damköhler, G. Einflüsse Der Strömung, Diffusion Und Des Wärmeüberganges Auf Die Leistung von Reaktionsöfen.: I. Allgemeine Gesichtspunkte Für Die Übertragung Eines Chemischen Prozesses Aus Dem Kleinen Ins Große. Z. Elektrochem. Angew. Phys. Chem. 1936, 42 (12), 846-862. 
(23) Sun, L.; Campbell, M. G.; Dincă, M. Electrically Conductive Porous Metal-Organic Frameworks. Angew. Chem., Int. Ed. 2016, 55 (11), 3566-3579.

(24) Jackson, M. N.; Oh, S.; Kaminsky, C. J.; Chu, S. B.; Zhang, G.; Miller, J. T.; Surendranath, Y. Strong Electronic Coupling of Molecular Sites to Graphitic Electrodes via Pyrazine Conjugation. J. Am. Chem. Soc. 2018, 140 (3), 1004-1010.

(25) Oh, S.; Gallagher, J. R.; Miller, J. T.; Surendranath, Y. GraphiteConjugated Rhenium Catalysts for Carbon Dioxide Reduction. J. Am. Chem. Soc. 2016, 138 (6), 1820-1823.

(26) Miner, E. M.; Fukushima, T.; Sheberla, D.; Sun, L.; Surendranath, Y.; Dincă, M. Electrochemical Oxygen Reduction Catalysed by Ni3(Hexaiminotriphenylene)2. Nat. Commun. 2016, 7 (1), 10942.

(27) Andrieux, C. P.; Savéant, J. M. Electron Transfer through Redox Polymer Films. J. Electroanal. Chem. Interfacial Electrochem. 1980, 111 (2-3), 377-381.

(28) Cai, M.; Loague, Q.; Morris, A. J. Design Rules for Efficient Charge Transfer in Metal-Organic Framework Films: The Pore Size Effect. J. Phys. Chem. Lett. 2020, 11, 702-709.

(29) Goswami, S.; Hod, I.; Duan, J. D.; Kung, C.-W.; Rimoldi, M.; Malliakas, C. D.; Palmer, R. H.; Farha, O. K.; Hupp, J. T. Anisotropic Redox Conductivity within a Metal-Organic Framework Material. J. Am. Chem. Soc. 2019, 141 (44), 17696-17702.

(30) Mohammad-Pour, G. S.; Hatfield, K. O.; Fairchild, D. C.; Hernandez-Burgos, K.; Rodríguez-López, J.; Uribe-Romo, F. J. A SolidSolution Approach for Redox Active Metal-Organic Frameworks with Tunable Redox Conductivity. J. Am. Chem. Soc. 2019, 141 (51), 19978-19982.

(31) Celis-Salazar, P. J.; Cai, M.; Cucinell, C. A.; Ahrenholtz, S. R.; Epley, C. C.; Usov, P. M.; Morris, A. J. Independent Quantification of Electron and Ion Diffusion in Metallocene-Doped Metal-Organic Frameworks Thin Films. J. Am. Chem. Soc. 2019, 141 (30), 1194711953.

(32) Roy, S.; Huang, Z.; Bhunia, A.; Castner, A.; Gupta, A. K.; Zou, X.; Ott, S. Electrocatalytic Hydrogen Evolution from a Cobaloxime-Based Metal-Organic Framework Thin Film. J. Am. Chem. Soc. 2019, 141 (40), 15942-15950.

(33) Matheu, R.; Gutierrez-Puebla, E.; Monge, M. Á.; Diercks, C. S.; Kang, J.; Prévot, M. S.; Pei, X.; Hanikel, N.; Zhang, B.; Yang, P.; Yaghi, O. M. Three-Dimensional Phthalocyanine Metal-Catecholates for High Electrochemical Carbon Dioxide Reduction. J. Am. Chem. Soc. 2019, 141 (43), 17081-17085.

(34) Johnson, B. A.; Bhunia, A.; Fei, H.; Cohen, S. M.; Ott, S. Development of a UiO-Type Thin Film Electrocatalysis Platform with Redox-Active Linkers. J. Am. Chem. Soc. 2018, 140 (8), 2985-2994.

(35) Dahms, H. Electronic Conduction in Aqueous Solution. J. Phys. Chem. 1968, 72 (1), 362-364.

(36) Ruff, I.; Friedrich, V. J. Transfer Diffusion. I. Theoretical. J. Phys. Chem. 1971, 75 (21), 3297-3302.

(37) Ruff, I.; Friedrich, V. J.; Demeter, K.; Csillag, K. Transfer Diffusion. II. Kinetics of Electron Exchange Reaction between Ferrocene and Ferricinium Ion in Alcohols. J. Phys. Chem. 1971, 75 (21), 3303-3309.

(38) Ruff, I.; Korosi-Odor, I. Application of Diffusion Constant Measurement to the Determination of the Rate Constant of ElectronExchange Reactions. Inorg. Chem. 1970, 9 (1), 186-188.

(39) Laviron, E. A Multilayer Model for the Study of Space Distributed Redox Modified Electrodes: Part I. Description and Discussion of the Model. J. Electroanal. Chem. Interfacial Electrochem. 1980, $112(1), 1-9$.

(40) Chidsey, C. E. D.; Murray, R. W. Redox Capacity and Direct Current Electron Conductivity in Electroactive Materials. J. Phys. Chem. 1986, 90 (7), 1479-1484.

(41) Buttry, D. A.; Anson, F. C. Electron Hopping vs. Molecular Diffusion as Charge Transfer Mechanisms in Redox Polymer Films. J. Electroanal. Chem. Interfacial Electrochem. 1981, 130 (C), 333-338.

(42) Peerce, P. J.; Bard, A. J. Polymer Films on Electrodes: Part III. Digital Simulation Model for Cyclic Voltammetry of Electroactive
Polymer Film and Electrochemistry of Poly(Vinylferrocene) on Platinum. J. Electroanal. Chem. Interfacial Electrochem. 1980, 114 (1), $89-115$.

(43) White, H. S.; Leddy, J.; Bard, A. J. Polymer Films on Electrodes. 8. Investigation of Charge-Transport Mechanisms in Nafion Polymer Modified Electrodes. J. Am. Chem. Soc. 1982, 104 (18), 4811-4817.

(44) Costentin, C.; Nocera, D. G. Dual-Phase Molecular-like Charge Transport in Nanoporous Transition Metal Oxides. J. Phys. Chem. C 2019, 123 (3), 1966-1973.

(45) Liberman, I.; Shimoni, R.; Ifraemov, R.; Rozenberg, I.; Singh, C.; Hod, I. Active-Site Modulation in an Fe-Porphyrin-Based MetalOrganic Framework through Ligand Axial Coordination: Accelerating Electrocatalysis and Charge-Transport Kinetics. J. Am. Chem. Soc. 2020, 142 (4), 1933-1940.

(46) Savéant, J. M. Electron Hopping between Fixed Sites: Equivalent Diffusion and Migration Laws. J. Electroanal. Chem. Interfacial Electrochem. 1986, 201 (1), 211-213.

(47) Savéant, J. M. Electron Hopping between Fixed Sites: "Diffusion" and "Migration" in Counter-Ion Conservative Redox Membranes at Steady State. J. Electroanal. Chem. Interfacial Electrochem. 1988, 242 (12), $1-21$.

(48) Andrieux, C. P.; Saveant, J. M. Electroneutrality Coupling of Electron Hopping between Localized Sites with Electroinactive Counterion Displacement. 1. Potential-Step Plateau Currents. J. Phys. Chem. 1988, 92 (23), 6761-6767.

(49) Hod, I.; Farha, O. K.; Hupp, J. T. Modulating the Rate of Charge Transport in a Metal-Organic Framework Thin Film Using Host:Guest Chemistry. Chem. Commun. 2016, 52 (8), 1705-1708.

(50) Ahrenholtz, S. R.; Epley, C. C.; Morris, A. J. Solvothermal Preparation of an Electrocatalytic Metalloporphyrin MOF Thin Film and Its Redox Hopping Charge-Transfer Mechanism. J. Am. Chem. Soc. 2014, 136 (6), 2464-2472.

(51) Lin, S.; Pineda-Galvan, Y.; Maza, W. A.; Epley, C. C.; Zhu, J.; Kessinger, M. C.; Pushkar, Y.; Morris, A. J. Electrochemical Water Oxidation by a Catalyst-Modified Metal-Organic Framework Thin Film. ChemSusChem 2017, 10 (3), 514-522.

(52) Saveant, J. M. Electron Hopping between Localized Sites: Effect of Ion Pairing on Diffusion and Migration; General Rate Laws and Steady-State Responses. J. Phys. Chem. 1988, 92 (15), 4526-4532.

(53) Anson, F. C.; Blauch, D. N.; Savéant, J. M.; Shu, C. F. Ion Association and Electric Field Effects on Electron Hopping in Redox Polymers. Application to the Tris(2,2'-Bipyridine)Osmium(3+)/Tris(2,2'-Bipyridine)Osmium(2+) Couple in Nafion. J. Am. Chem. Soc. 1991, 113 (6), 1922-1932.

(54) Lin, S.; Usov, P. M.; Morris, A. J. The Role of Redox Hopping in Metal-Organic Framework Electrocatalysis. Chem. Commun. 2018, 54 (51), 6965-6974.

(55) Aoki, K.; Tokuda, K.; Matsuda, H. Theory of Linear Sweep Voltammetry with Finite Diffusion Space. J. Electroanal. Chem. Interfacial Electrochem. 1983, 146 (2), 417-424.

(56) Aoki, K.; Tokuda, K.; Matsuda, H. Theory of Linear Sweep Voltammetry with Finite Diffusion Space: Part II. Totally Irreversible and Quasi-Reversible Cases. J. Electroanal. J. Electroanal. Chem. Interfacial Electrochem. 1984, 160 (1), 33-45.

(57) Peerce, P. J.; Bard, A. J. Polymer Films on Electrodes. J. Electroanal. Chem. Interfacial Electrochem. 1980, 114 (1), 89-115.

(58) Costentin, C.; Porter, T. R.; Savéant, J.-M. How Do Pseudocapacitors Store Energy? Theoretical Analysis and Experimental Illustration. ACS Appl. Mater. Interfaces 2017, 9 (10), 8649-8658.

(59) Buesen, D.; Li, H.; Plumeré, N. The Electron as a Probe to Measure the Thickness Distributions of Electroactive Films. Chem. Sci. 2020, 11 (4), 937-946.

(60) Li, H.; Buesen, D.; Williams, R.; Henig, J.; Stapf, S.; Mukherjee, K.; Freier, E.; Lubitz, W.; Winkler, M.; Happe, T.; Plumeré, N. Preventing the Coffee-Ring Effect and Aggregate Sedimentation by in Situ Gelation of Monodisperse Materials. Chem. Sci. 2018, 9 (39), $7596-7605$.

(61) Andrieux, C. P.; Dumas-Bouchiat, J. M.; Savéant, J. M. Catalysis of Electrochemical Reactions at Redox Polymer Electrodes. Kinetic 
Model for Stationary Voltammetric Techniques. J. Electroanal. Chem. Interfacial Electrochem. 1982, 131 (C), 1-35.

(62) Andrieux, C. P.; Savéant, J. M. Kinetics of Electrochemical Reactions Mediated by Redox Polymer Films: Irreversible CrossExchange Reactions: Formulation in Terms of Characteristic Currents for Stationary Techniques. J. Electroanal. Chem. Interfacial Electrochem. 1982, 134 (1), 163-166.

(63) Andrieux, C. P.; Dumas-Bouchiat, J. M.; Savéant, J. M. Kinetics of Electrochemical Reactions Mediated by Redox Polymer Films. New Formulation and Strategies for Analysis and Optimization. J. Electroanal. Chem. Interfacial Electrochem. 1984, 169 (1-2), 9-21.

(64) Bartlett, P. N.; Pratt, K. F. E. Theoretical Treatment of Diffusion and Kinetics in Amperometric Immobilized Enzyme Electrodes Part I: Redox Mediator Entrapped within the Film. J. Electroanal. Chem. 1995, 397 (1-2), 61-78.

(65) Fourmond, V.; Stapf, S.; Li, H.; Buesen, D.; Birrell, J.; Rüdiger, O.; Lubitz, W.; Schuhmann, W.; Plumeré, N.; Léger, C. Mechanism of Protection of Catalysts Supported in Redox Hydrogel Films. J. Am. Chem. Soc. 2015, 137 (16), 5494-5505.

(66) Li, H.; Buesen, D.; Dementin, S.; Léger, C.; Fourmond, V.; Plumeré, N. Complete Protection of O2-Sensitive Catalysts in Thin Films. J. Am. Chem. Soc. 2019, 141 (42), 16734-16742.

(67) Costentin, C.; Savéant, J.-M. Cyclic Voltammetry Analysis of Electrocatalytic Films. J. Phys. Chem. C 2015, 119 (22), 12174-12182.

(68) Costentin, C.; Savéant, J.-M. Cyclic Voltammetry of Electrocatalytic Films: Fast Catalysis Regimes. ChemElectroChem 2015, 2 (11), 1774-1784.

(69) Leddy, J.; Bard, A. J.; Maloy, J. T.; Savéant, J. M. Kinetics of FilmCoated Electrodes: Effect of a Finite Mass Transfer Rate of Substrate across the Film-Solution Interface at Steady State. J. Electroanal. Chem. Interfacial Electrochem. 1985, 187 (2), 205-227.

(70) Savéant, J.-M. Permeation through Polymer Coatings on Electrodes: Membrane versus Pinhole Permeation. J. Electroanal. Chem. Interfacial Electrochem. 1991, 302 (1-2), 91-101.

(71) Ewing, A. G.; Feldman, B. J.; Murray, R. W. Permeation of Neutral, Cationic, and Anionic Electrode Reactants through a Polycationic Polymer Film as a Function of Electrolyte Concentration. J. Phys. Chem. 1985, 89 (7), 1263-1269.

(72) Wang, C.; Wang, J.-L.; Lin, W. Elucidating Molecular Iridium Water Oxidation Catalysts Using Metal-Organic Frameworks: A Comprehensive Structural, Catalytic, Spectroscopic, and Kinetic Study. J. Am. Chem. Soc. 2012, 134 (48), 19895-19908.

(73) Andrieux, C. P.; Dumas-Bouchiat, J. M.; Savéant, J. M. Catalysis of Electrochemical Reactions at Redox Polymer Electrodes. J. Electroanal. Chem. Interfacial Electrochem. 1980, 114 (1), 159-163.

(74) Andrieux, C. P.; Saveant, J. M. Heterogeneous (Chemically Modified Electrodes, Polymer Electrodes) vs. Homogeneous Catalysis of Electrochemical Reactions. J. Electroanal. Chem. Interfacial Electrochem. 1978, 93 (2), 163-168.

(75) Rocklin, R. D.; Murray, R. W. Kinetics of Electrocatalysis of Dibromoalkyl Reductions Using Electrodes with Covalently Immobilized Metallotetraphenylporphyrins. J. Phys. Chem. 1981, 85 (14), 2104-2112.

(76) Costentin, C.; Savéant, J.-M. Cyclic Voltammetry of Fast Conducting Electrocatalytic Films. Phys. Chem. Chem. Phys. 2015, 17 (29), 19350-19359.

(77) Costentin, C.; Savéant, J.-M. Elements of Molecular and Biomolecular Electrochemistry, 2nd ed.; John Wiley \& Sons, Inc.: Hoboken, NJ, 2019.

(78) Bard, A. J.; Faulkner, L. R. Electrochemical Methods: Fundamental and Applications, 2nd ed.; John Wiley \& Sons, Inc.: Hoboken, NJ, 2001.

(79) Rudolph, M. A Fast Implicit Finite Difference Algorithm for the Digital Simulation of Electrochemical Processes. J. Electroanal. Chem. Interfacial Electrochem. 1991, 314 (1-2), 13-22.

(80) Britz, D.; Strutwolf, J. Digital Simulation in Electrochemistry, 4th ed.; Springer International Publishing, 2016.

(81) Andrieux, C. P.; Savéant, J. M. Catalysis at Redox Polymer Coated Electrodes. In Molecular Design of Electrode Surfaces;
Techniques of Chemistry 22; Murray, R. W., Ed.; John Wiley \& Sons, Inc., 1992; pp 207-270.

(82) McCarthy, B. D.; Beiler, A. M.; Johnson, B. A.; Liseev, T.; Castner, A. T.; Ott, S. Analysis of Electrocatalytic Metal-Organic Frameworks. Coord. Chem. Rev. 2020, 406, 213137.

(83) Kornienko, N.; Zhao, Y.; Kley, C. S.; Zhu, C.; Kim, D.; Lin, S.; Chang, C. J.; Yaghi, O. M.; Yang, P. Metal-Organic Frameworks for Electrocatalytic Reduction of Carbon Dioxide. J. Am. Chem. Soc. 2015, 137 (44), 14129-14135.

(84) Johnson, B. A.; Bhunia, A.; Ott, S. Electrocatalytic Water Oxidation by a Molecular Catalyst Incorporated into a Metal-Organic Framework Thin Film. Dalt. Trans. 2017, 46 (5), 1382-1388.

(85) McCrory, C. C. L.; Jung, S.; Ferrer, I. M.; Chatman, S. M.; Peters, J. C.; Jaramillo, T. F. Benchmarking Hydrogen Evolving Reaction and Oxygen Evolving Reaction Electrocatalysts for Solar Water Splitting Devices. J. Am. Chem. Soc. 2015, 137 (13), 4347-4357.

(86) Wang, C.; Lin, W. Diffusion-Controlled Luminescence Quenching in Metal-Organic Frameworks. J. Am. Chem. Soc. 2011, 133 (12), 4232-4235.

(87) Shekhah, O.; Liu, J.; Fischer, R. A.; Wöll, C. MOF Thin Films: Existing and Future Applications. Chem. Soc. Rev. 2011, 40 (2), 1081.

(88) Bétard, A.; Fischer, R. A. Metal-Organic Framework Thin Films: From Fundamentals to Applications. Chem. Rev. 2012, 112 (2), 10551083.

(89) Yaghi, O. M. Reticular Chemistry in All Dimensions. ACS Cent. Sci. 2019, 5 (8), 1295-1300.

(90) Yaghi, O. M. Reticular Chemistry: Molecular Precision in Infinite 2D and 3D. Mol. Front. J. 2019, 03 (01), 66-83. 